\title{
RESIDUE CURRENTS WITH PRESCRIBED ANNIHILATOR IDEALS
}

\author{
By MATS ANDERSSON ${ }^{1}$ AND ELIZABETH WULCAN
}

ABSTRACT. - Given a coherent ideal sheaf $J$ we construct locally a vector-valued residue current $R$ whose annihilator is precisely the given sheaf. In case $J$ is a complete intersection, $R$ is just the classical Coleff-Herrera product. By means of these currents we can extend various results, previously known for a complete intersection, to general ideal sheaves. Combining with integral formulas we obtain a residue version of the Ehrenpreis-Palamodov fundamental principle.

(C) 2007 Elsevier Masson SAS

RÉSUMÉ. - Soit $\mathcal{J}$ un faisceau cohérent d'idéaux. Nous construisons localement un courant résiduel $R$ à valeurs vectorielles dont l'annihilateur est $\mathcal{J}$. Au cas où $\mathcal{J}$ serait une intersection complète, $R$ est simplement le produit classique de Coleff-Herrera. Ces courants permettent d'étendre au cas général des résultats divers, déjà connus dans le cas d'une intersection complète. En utilisant ces courants résiduels et des formules intégrales, nous obtenons ainsi une version résiduelle du Principe Fondamental d'EhrenpreisPalamodov.

(C) 2007 Elsevier Masson SAS

\section{Introduction}

Let $h=h_{1}, \ldots, h_{m}$ be a tuple of holomorphic functions such that their common zero set $Z$ has codimension $m$, and let

$$
\mu^{h}=\bar{\partial} \frac{1}{h_{1}} \wedge \cdots \wedge \bar{\partial} \frac{1}{h_{m}}
$$

be the Coleff-Herrera product introduced in [16]. Dickenstein and Sessa, [18], and Passare, [31], independently proved the duality principle, that a holomorphic function $\phi$ is in the ideal sheaf $\mathcal{J}(h)$ generated by $h_{1}, \ldots, h_{m}$ if and only if the current $\phi \mu^{h}$ vanishes, i.e., $\phi$ belongs to the annihilator ann $\mu^{h}$. Given any coherent ideal sheaf $\mathcal{J}$ one can locally find a finite tuple $\gamma=\left(\gamma_{1}, \ldots, \gamma_{\mu}\right)$ of so-called Coleff-Herrera currents such that $\mathcal{J}=\operatorname{ann} \gamma=\bigcap_{j}$ ann $\gamma_{j}$; this is closely related to the existence of Noetherian operators, see [15]. However, much of the utility of the duality principle depends on the fact that the current $\mu^{h}$ fits into various division-interpolation integral formulas, see, e.g., $[13,31,14,11,12]$. Therefore it is natural to look for an analogue, for a general ideal sheaf, with this extra property.

\footnotetext{
${ }^{1}$ The first author was partially supported by the Swedish Natural Science Research Council. 
To begin with we consider an arbitrary complex of Hermitian holomorphic vector bundles over a complex manifold $X$,

$$
0 \rightarrow E_{N} \stackrel{f_{N}}{\longrightarrow} \cdots \stackrel{f_{3}}{\longrightarrow} E_{2} \stackrel{f_{2}}{\longrightarrow} E_{1} \stackrel{f_{1}}{\longrightarrow} E_{0}
$$

that is exact outside an analytic variety $Z$ of positive codimension. To this complex $E_{\bullet}$ we associate a current $R=R\left(E_{\bullet}\right)$ taking values in $\operatorname{End}\left(\bigoplus_{k} E_{k}\right)$ and with support on $Z$. This current in a certain way measures the lack of exactness of the associated complex of locally free sheaves of $\mathcal{O}$-modules of sections of $E_{k}$

$$
0 \rightarrow \mathcal{O}\left(E_{N}\right) \rightarrow \cdots \rightarrow \mathcal{O}\left(E_{1}\right) \rightarrow \mathcal{O}\left(E_{0}\right)
$$

Let $R^{\ell}$ denote the component of $R$ that takes values in $\operatorname{Hom}\left(E_{\ell}, \bigoplus_{k} E_{k}\right)$. It turns out that (1.3) is exact if and only if $R^{\ell}=0$ for $\ell \geqslant 1$ (Theorem 3.1). Let $\mathcal{J}=\operatorname{Im}\left(\mathcal{O}\left(E_{1}\right) \rightarrow \mathcal{O}\left(E_{0}\right)\right)$. The main result in this paper is the following:

THEOREM 1.1. - Suppose that the sheaf complex (1.3) is exact. Then the associated residue current $R$ has its support on the set $Z$ where the sheaf $\mathcal{O}\left(E_{0}\right) / \mathcal{J}$ is not locally free, and a local holomorphic section $\phi$ of $E_{0}$ is in $\mathcal{J}$ if and only if $\phi$ is generically in the image of $f_{1}$ and the residue current $R \phi$ vanishes.

The set $Z$ is precisely the set where the mapping $f_{1}$ does not have optimal rank. If $f_{1}$ is generically surjective, or equivalently $\operatorname{ann}\left(\mathcal{O}\left(E_{0}\right) / \mathcal{J}\right)$ is nonzero, thus $\phi \in \mathcal{J}$ if and only if $R \phi=0$. In this case $Z$ is the zero locus of $\operatorname{ann}\left(\mathcal{O}\left(E_{0}\right) / \mathcal{J}\right)$. In particular as soon as $\mathcal{J}$ is a nontrivial ideal sheaf (rank $E_{0}=1$ ) then $R$ has its support on the zero locus of $\mathcal{J}$ and $\phi \in \mathcal{J}$ if and only if $R \phi=0$. In analogy with Noetherian differential operators it is natural to say that $R$ is a Noetherian residue current for $\mathcal{J}$.

If $\mathcal{J}$ is any coherent subsheaf of some locally free sheaf $\mathcal{O}\left(E_{0}\right)$, then at least locally $\mathcal{O}\left(E_{0}\right) / \mathcal{J}$ admits a resolution (1.3), and if we equip the corresponding complex of vector bundles with any Hermitian metric we thus locally get a current $R$ as in Theorem 1.1. In case $\mathcal{J}$ is defined by a complete intersection, the Koszul complex provides a resolution, and the resulting residue current is just the Coleff-Herrera product, see Example 1 below. In general it is just as hard to find resolutions of ideals as to find, e.g., Noetherian differential operators, so Theorem 1.1 will not contribute to effectivity questions, but it turns out to be useful in several other ways.

If $\mathcal{O}\left(E_{0}\right) / \mathcal{J}$ is a sheaf of Cohen-Macaulay modules, the associated current $R$ is independent of the Hermitian metrics and it is essentially canonical, see Section 4 for precise statements. In the Cohen-Macaulay case we can also define a cohomological residue for $\mathcal{J}$, so that the cohomological duality principle for a complete intersection ideal extends (Theorem 4.2).

Combined with the framework of integral formulas developed in [5], we present in Section 5 a holomorphic division formula, (5.4), for sections of $E_{k}$. In particular, as soon as $\phi \in \mathcal{J}$, this formula provides an explicit realization of the membership. By a similar integral formula we obtain a residue characterization (Theorem 5.1) of the sheaf $\mathcal{E} \mathcal{J}$ of $\mathcal{E}$-modules generated by $\mathcal{J}$.

Given a module $J$ over $\mathbb{C}\left[z_{1}, \ldots, z_{n}\right]$, generated by an $r_{0} \times r_{1}$-matrix $F(z)$ of polynomials in $\mathbb{C}^{n}$ of generic rank $r_{0}$ we can find a global Noetherian residue current $R$ for the corresponding sheaf $\mathcal{J}$ in $\mathbb{C}^{n}$. It is obtained from a resolution of the module over the graded ring $\mathbb{C}\left[z_{0}, \ldots, z_{n}\right]$ induced by a homogenization of $F$. We can use this current to prove a generalization of Max Noether's classical $A F+B G$ theorem. Our main application is a residue version of the general fundamental principle: If $F^{T}$ is the transpose of $F$, then any smooth solution to $F^{T}(i \partial / \partial t) \xi=0$

$4^{\mathrm{e}}$ SÉRIE - TOME $40-2007-\mathrm{N}^{\circ} 6$ 
on a smoothly bounded convex set in $\mathbb{R}^{n}$ can be written

$$
\xi(t)=\int_{\mathbb{C}^{n}} R^{T}(\zeta) A(\zeta) e^{-i\langle t, \zeta\rangle},
$$

for an appropriate (explicitly given matrix of smooth functions) $A$; here $R^{T}$ is the transpose of $R$. Conversely, since $R$ is Noetherian, any $\xi(t)$ given in this way is a homogeneous solution. This follows along the same lines as in [14], where this result was obtained for a complete intersection $F$ by means of the Coleff-Herrera product.

Throughout this paper, $\mathcal{E}_{\bullet}(E), \mathcal{D}_{\bullet}(E), \mathcal{D}_{\bullet}^{\prime}(E)$, and $\mathcal{O}(E)$ denote the sheaves of smooth forms, test forms, currents, and holomorphic functions, respectively with values in the vector bundle $E$.

\section{Residue currents of generically exact complexes}

Let $E, Q$ be Hermitian holomorphic vector bundles over a connected manifold $X$ and let $f: E \rightarrow Q$ be a holomorphic morphism. If $f$ has optimal rank $\rho$ then the rank is precisely $\rho$ outside the analytic set $Z=\{F=0\}$, where $F=\operatorname{det}^{\rho} f$ is a section of $\Lambda^{\rho} E^{*} \otimes \Lambda^{\rho} Q$. Let $\sigma: Q \rightarrow E$ be the minimal inverse in $X \backslash Z$, i.e., $\sigma \xi$ is the minimal solution to $f \eta=\xi$ if $\xi$ is in the image of $f$ and $\sigma \xi=0$ if $\xi$ is orthogonal to $\operatorname{Im} f$. Then clearly $\sigma$ is smooth outside $Z$, and following the proof of Lemma 4.1 in [4] we get

LEMMA 2.1. - If $F=F^{0} F^{\prime}$ in $X$, where $F^{0}$ is a holomorphic function and $F^{\prime}$ is nonvanishing, then $F^{0} \sigma$ is smooth across $Z$.

Let

$$
0 \rightarrow E_{N} \stackrel{f_{N}}{\longrightarrow} E_{N-1} \stackrel{f_{N-1}}{\longrightarrow} \cdots \stackrel{f_{-M+2}}{\longrightarrow} E_{-M+1} \stackrel{f_{-M+1}}{\longrightarrow} E_{-M} \rightarrow 0
$$

be a holomorphic complex of Hermitian vector bundles over the $n$-dimensional complex manifold $X$, and assume that it is pointwise exact outside the analytic set $Z$ of positive codimension. Then for each $k$, $\operatorname{rank} f_{k}$ is constant in $X \backslash Z$ and equal to

$$
\rho_{k}=\operatorname{dim} E_{k}-\operatorname{dim} E_{k+1}+\cdots \pm \operatorname{dim} E_{N} .
$$

The bundle $E=\bigoplus E_{k}$ has a natural superbundle structure, i.e., a $\mathbb{Z}_{2}$-grading, $E=E^{+} \oplus E^{-}$, $E^{+}$and $E^{-}$being the subspaces of even and odd elements, respectively, by letting $E^{+}=$ $\bigoplus_{2 k} E_{k}$ and $E^{-}=\bigoplus_{2 k+1} E_{k}$, see [34] and, e.g., [5], for details. The mappings $f=\sum f_{j}$ and $\bar{\partial}$ are then odd mappings on $\mathcal{D}_{\bullet}^{\prime}(E)$ and they anticommute so that $\nabla^{2}=0$, where $\nabla=f-\bar{\partial}$ is (minus) the $(0,1)$-part of Quillen's superconnection $D-\bar{\partial}$. Moreover, $\nabla$ extends to an odd mapping $\nabla_{\text {End }}$ on $\mathcal{D}_{\bullet}^{\prime}\left(\right.$ End $E$ ) and $\nabla_{\text {End }}^{2}=0$. In $X \backslash Z$ let $\sigma_{k}: E_{k-1} \rightarrow E_{k}$ be the minimal inverses of $f_{k}$. If $\sigma=\sigma_{-M+1}+\cdots+\sigma_{N}: E \rightarrow E$ and $I$ denotes the identity endomorphism on $E$, then $f \sigma+\sigma f=I$. Moreover, $\sigma \sigma=0$ and thus

$$
\sigma(\bar{\partial} \sigma)=(\bar{\partial} \sigma) \sigma
$$

Since $\sigma$ is odd, $\nabla_{\text {End }} \sigma=\nabla \circ \sigma+\sigma \circ \nabla=f \sigma+\sigma f-(\bar{\partial} \circ \sigma+\sigma \circ \bar{\partial})$, so we get

$$
\nabla_{\text {End }} \sigma=I-\bar{\partial} \sigma .
$$

Notice that $\bar{\partial} \sigma$ has even degree. In $X \backslash Z$ we define the End $E$-valued form, cf. (2.4),

$$
u=\sigma\left(\nabla_{\mathrm{End}} \sigma\right)^{-1}=\sigma(I-\bar{\partial} \sigma)^{-1}=\sigma+\sigma(\bar{\partial} \sigma)+\sigma(\bar{\partial} \sigma)^{2}+\cdots
$$


Now, $\nabla_{\text {End }} u=\nabla_{\text {End }} \sigma\left(\nabla_{\text {End }} \sigma\right)^{-1}-\sigma \nabla_{\text {End }}\left(\nabla_{\text {End }} \sigma\right)^{-1}$, and since $\nabla_{\text {End }}^{2}=0$ we thus have

$$
\nabla_{\text {End }} u=I \text {. }
$$

Notice that

$$
u=\sum_{\ell} \sum_{k \geqslant \ell+1} u_{k}^{\ell}
$$

where

$$
u_{k}^{\ell}=\sigma_{k}\left(\bar{\partial} \sigma_{k-1}\right) \cdots\left(\bar{\partial} \sigma_{\ell+1}\right)
$$

is in $\mathcal{E}_{0, k-\ell-1}\left(\operatorname{Hom}\left(E_{\ell}, E_{k}\right)\right)$ over $X \backslash Z$. In view of (2.3) we also have

$$
u_{k}^{\ell}=\left(\bar{\partial} \sigma_{k}\right)\left(\bar{\partial} \sigma_{k-1}\right) \cdots\left(\bar{\partial} \sigma_{\ell+2}\right) \sigma_{\ell+1} .
$$

Let

$$
u^{\ell}=\sum_{k \geqslant \ell+1} u_{k}^{\ell}
$$

be $u$ composed with the projection $E \rightarrow E_{\ell}$. We can make a current extension of $u$ across $Z$ following [33] and the proof of Theorem 1.1 in [1]. In fact, after a sequence of suitable resolutions we may assume that the sections $F_{j}=\operatorname{det}^{\rho_{j}} f_{j}$ of $\Lambda^{\rho_{j}} E_{j}^{*} \otimes \Lambda^{\rho_{j}} E_{j-1}$ are of the form $F_{j}=F_{j}^{0} F_{j}^{\prime}$, where $F_{j}^{0}$ is a monomial and $F_{j}^{\prime}$ are non-vanishing. If $F$ is a holomorphic function that vanishes on $Z$, in the same way we may assume that $F=F^{0} F^{\prime}$. By Lemma $2.1, \sigma_{j}=\alpha_{j} / F_{j}^{0}$, where $\alpha_{j}$ is smooth across $Z$. Since $\alpha_{j+1} \alpha_{j}=0$ outside the set $\left\{F_{j+1}^{0} F_{j}^{0}=0\right\}$, thus $\alpha_{j+1} \alpha_{j}=0$ everywhere. Therefore, cf. (2.7), it is easy to see that

$$
u_{\ell+k}^{\ell}=\frac{\left(\bar{\partial} \alpha_{\ell+k}\right)\left(\bar{\partial} \alpha_{\ell+k-1}\right) \cdots\left(\bar{\partial} \alpha_{\ell+2}\right) \alpha_{\ell+1}}{F_{\ell+k}^{0} \cdots F_{\ell+1}^{0}} .
$$

Since $F_{j}$ only vanish on $Z$ and $F$ vanishes there, $F^{0}$ must contain each coordinate factor that occurs in any $F_{j}^{0}$. It follows now that $\lambda \mapsto|F|^{2 \lambda} u$ has a current-valued analytic continuation to $\operatorname{Re} \lambda>-\epsilon$, and that $U=\left.|F|^{2 \lambda} u\right|_{\lambda=0}$ is a current extension of $u$.

In the same way we can now define the residue current $R=R\left(E_{\bullet}\right)$ associated to (2.1) as

$$
R=\left.\bar{\partial}|F|^{2 \lambda} \wedge u\right|_{\lambda=0}
$$

It clearly has its support on $Z$. If $R_{k}^{\ell}=\left.\bar{\partial}|F|^{2 \lambda} \wedge u_{k}^{\ell}\right|_{\lambda=0}$ and $R^{\ell}$ is defined analogously, then

$$
R=\sum_{\ell} R^{\ell}=\sum_{\ell} \sum_{k \geqslant \ell+1} R_{k}^{\ell}
$$

Notice that $R_{k}^{\ell}$ is a $\operatorname{Hom}\left(E_{\ell}, E_{k}\right)$-valued $(0, k-\ell)$-current. The currents $U^{\ell}$ and $U_{k}^{\ell}$ are defined analogously. Notice that $U$ has odd degree and $R$ has even degree. In analogy with Theorems 1.1 and 1.2 in [1] we have:

PROPOSITION 2.2. - If $U$ and $R$ are the currents associated to the complex (2.1) then

$$
\nabla_{\text {End }} U=I-R, \quad \nabla_{\text {End }} R=0 .
$$

$4^{\text {e }}$ SÉRIE - TOME $40-2007-\mathrm{N}^{\circ} 6$ 
Moreover, $R_{k}^{\ell}$ vanishes if $k-\ell<\operatorname{codim} Z$, and $\bar{\xi} R=d \bar{\xi} \wedge R=0$ if $\xi$ is holomorphic and vanishes on $Z$.

The residue current $R=R\left(E_{\bullet}\right)$ is related to the (lack of) exactness of the sheaf complex associated to (2.1) in the following way.

PROposition 2.3. - Let $R=R\left(E_{\bullet}\right)$ be the residue current associated with (2.1) and let $\phi$ be a holomorphic section of $E_{\ell}$.

(i) If $f_{\ell} \phi=0$ and $R^{\ell} \phi=0$, then locally there is a holomorphic section $\psi$ of $E_{\ell+1}$ such that $f_{\ell+1} \psi=\phi$.

(ii) If moreover $R^{\ell+1}=0$, then the existence of such a local solution $\psi$ implies that $R^{\ell} \phi=0$.

Proof. - Let $U$ be the associated current such that (2.9) holds. Then $\nabla(U \phi)=$ $\phi-U(\nabla \phi)-R \phi$. Since $U \phi=U^{\ell} \phi, R \phi=R^{\ell} \phi$, and $\nabla \phi=f_{\ell} \phi-\bar{\partial} \phi$, it follows from the assumptions of $\phi$ that $\nabla\left(U^{\ell} \phi\right)=\phi$. Now (i) follows by solving a sequence of $\bar{\partial}$-equations locally. For the second part, assume that $f_{\ell+1} \psi=\phi$. Then by (2.9), $R^{\ell} \phi=R \phi=R(\nabla \psi)=\nabla(R \psi)=$ $\nabla\left(R^{\ell+1} \psi\right)=0$.

If now (1.2) is a generically exact holomorphic complex of Hermitian bundles, since rank $f_{1}$ is generically constant, we can define $\sigma_{1}$ in an unambiguous way in $X \backslash Z$, and therefore the currents $R^{\ell}$ for $\ell \geqslant 0$ can be defined as above, and we have:

COROLlaRY 2.4. - If $R=R\left(E_{\bullet}\right)$ is the residue current associated to (1.2), then Proposition 2.3 holds (for $\ell \geqslant 0$ ), provided that $f_{0} \phi=0$ is interpreted as $\phi$ belonging generically (outside $Z$ ) to the image of $f_{1}$.

If $f_{1}$ is generically surjective, in particular if rank $E_{0}=1$ and $f_{1}$ is not identically 0 , then this latter condition is of course automatically fulfilled.

Proof. - The corollary actually follows just from a careful inspection of the arguments in the proof of Proposition 2.3. Another way is to extend (1.2) to a generically exact complex (2.1) and then refer directly to Proposition 2.3, noting that the definition of $R^{\ell}$ for $\ell \geqslant 0$ as well as the condition $f_{0} \phi=0$ are independent of such an extension.

\section{Residue currents with prescribed annihilators}

The exactness of (1.3) is characterized by the current $R$ associated with (1.2).

THEOREM 3.1. - Assume that (1.2) is generically exact, let $R$ be the associated residue current, and let (1.3) be the associated complex of sheaves. Then $R^{\ell}=0$ for all $\ell \geqslant 1$ if and only if (1.3) is exact.

For the proof we will use the following characterization of exactness due to Buchsbaum and Eisenbud, see [21] Theorem 20.9: The complex (1.3) is exact if and only if

$$
\operatorname{codim} Z_{j} \geqslant j
$$

for all $j$, where, cf. (2.2),

$$
Z_{j}=\left\{z ; \operatorname{rank} f_{j}<\rho_{j}\right\} .
$$

Remark 1. - To be precise we will only use the "only if"-direction. The other direction is actually a consequence of Corollary 2.4 and (the proof of) Theorem 3.1. 
Proof. - From Corollary 2.4 it follows that (1.3) is exact if $R^{\ell}=0$ for $\ell \geqslant 1$. For the converse, let us now assume that (1.3) is exact; by the Buchsbaum-Eisenbud theorem then (3.1) holds. We will prove that $R^{1}=0$; the case when $\ell>1$ is handled in the same way. The idea in the proof is based on the somewhat vague principle that a residue current of bidegree $(0, q)$ cannot be supported on a variety of codimension $q+1$. Taking this for granted, we notice to begin with that $R_{2}^{1}=\left.\bar{\partial}|F|^{2 \lambda} \wedge \sigma_{2}\right|_{\lambda=0}$ is a $(0,1)$-current and has its support on $Z_{2}$, which has codimension at least 2 . Hence $R_{2}^{1}$ must vanish according to the vague principle. Now, $\sigma_{3}$ is smooth outside $Z_{3}$, and hence $R_{3}^{1}=\bar{\partial} \sigma_{3} \wedge R_{2}^{1}=0$ outside $Z_{3}$; thus $R_{3}^{1}$ is supported on $Z_{3}$ and again, by the same principle, $R_{3}^{1}$ must vanish etc. To make this into a strict argument we will use the following simple lemma which follows from a Taylor expansion.

Lemma 3.2. - Suppose that $\gamma(s, \tau)$ is smooth in $\mathbb{C} \times \mathbb{C}^{r}$ and that moreover $\gamma(s, \tau) / \bar{s}$ is smooth where $\tau_{1} \cdots \tau_{k} \neq 0$. Then $\gamma(s, \tau) / \bar{s}$ is smooth everywhere.

After a sequence of resolutions of singularities the action of $R_{k}^{1}$ on a test form $\xi$ is a finite sum of integrals of the form

$$
\left.\int \bar{\partial}\left|F^{0}\right|^{2 \lambda} \wedge \frac{\left(\bar{\partial} \alpha_{k}\right)\left(\bar{\partial} \alpha_{k-1}\right) \cdots\left(\bar{\partial} \alpha_{3}\right) \alpha_{2}}{F_{k}^{0} F_{k-1}^{0} \cdots F_{3}^{0} F_{2}^{0}} \wedge \tilde{\xi}\right|_{\lambda=0}
$$

where $F^{0}, F_{i}^{0}$ and $\alpha_{i}$ are as (2.8) above, and where $\tilde{\xi}$ is the pullback of $\xi$. To be precise, there are also cutoff functions involved that we suppress for simplicity. Observe that $\bar{\partial}\left|F^{0}\right|^{2 \lambda}$ is a finite sum of terms like $a \lambda\left|F^{0}\right|^{2 \lambda} d \bar{s} / \bar{s}$, where $a$ is a positive integer and $s$ is just one of the coordinate functions that divide $F^{0}$. We need to show that all the corresponding integrals vanish when $\lambda=0$, and to this end it is enough to show, see, e.g., Lemma 2.1 in [1], that

$$
\eta=\frac{d \bar{s}}{\bar{s}} \wedge\left(\bar{\partial} \alpha_{k}\right)\left(\bar{\partial} \alpha_{k-1}\right) \cdots\left(\bar{\partial} \alpha_{3}\right) \alpha_{2} \wedge \tilde{\xi}
$$

is smooth $((d \bar{s} / \bar{s}) \wedge \beta$ being smooth for a smooth $\beta$ means that each term of $\beta$ contains a factor $\bar{s}$ or $d \bar{s})$.

Let $\ell$ be the largest index among $2, \ldots, k$ such that $s$ is a factor in $F_{\ell}^{0}$ (possibly there is no such index at all; then $\ell$ below is to be interpreted as 1) and let $\tau_{1}, \ldots, \tau_{r}$ denote the coordinates that divide $F_{k}^{0} \cdots F_{\ell+1}^{0}$. We claim that, outside $\tau_{1} \cdots \tau_{r}=0$, the form

$$
\frac{d \bar{s}}{\bar{s}} \wedge \frac{\left(\bar{\partial} \alpha_{k}\right) \cdots\left(\bar{\partial} \alpha_{\ell+1}\right)}{F_{k}^{0} \cdots F_{\ell+1}^{0}} \wedge \tilde{\xi}
$$

is smooth. This follows by standard arguments, see, e.g., the proof of Lemma 2.2 in [33] or the proof of Theorem 1.1 in [1]; in fact, outside $Z_{k} \cap \cdots \cap Z_{\ell+1}$ the $(n, n-\ell+1)$-form $\left(\bar{\partial} \sigma_{k}\right) \cdots\left(\bar{\partial} \sigma_{\ell+1}\right) \wedge \xi$ is smooth and it must vanish on $Z_{\ell}$ for degree reasons, since $Z_{\ell}$ has codimension at least $\ell$. Thus the form

$$
\tilde{\eta}=\frac{d \bar{s}}{\bar{s}} \wedge\left(\bar{\partial} \alpha_{k}\right) \cdots\left(\bar{\partial} \alpha_{\ell+1}\right) \wedge \tilde{\xi}
$$

is smooth outside $\tau_{1} \cdots \tau_{r}=0$. By Lemma 3.2, applied to

$$
\gamma=d \bar{s} \wedge\left(\bar{\partial} \alpha_{k}\right) \cdots\left(\bar{\partial} \alpha_{\ell+1}\right) \wedge \tilde{\xi}
$$

$\tilde{\eta}$ is smooth everywhere, and therefore $\eta$ is smooth.

$4^{\text {e }}$ SÉRIE - TOME $40-2007-\mathrm{N}^{\circ} 6$ 
If (1.3) is exact, then, with no ambiguity, we can write $R_{k}$ rather than $R_{k}^{0}$.

Proof of Theorem 1.1. - Since a free resolution of a free sheaf is pointwise exact, it follows that $Z_{N} \subset \cdots \subset Z_{1}=Z$. Therefore $u^{0}$ is smooth outside $Z$ and thus the support of $R$ must be contained in $Z$. By Theorem 3.1, $R^{1}=0$, and so the second assertion, the Noetherian property of $R=R^{0}$, follows from Corollary 2.4.

Given any coherent sheaf $\mathcal{F}$ in a Stein manifold $X$ and compact subset $K \subset X$, one can always find a resolution

$$
\cdots \rightarrow \mathcal{O}^{\oplus r_{2}} \rightarrow \mathcal{O}^{\oplus r_{1}} \rightarrow \mathcal{O}^{\oplus r_{0}}
$$

of $\mathcal{F}$ in a neighborhood of $K$, e.g., by iterated use of Theorem 7.2.1 in [25]. The key stone in the proof of Theorem 3.1, the Buchsbaum-Eisenbud theorem, in general requires that the resolution (3.2) starts with 0 somewhere on the left. However, by the Syzygy theorem and Oka's lemma, $\operatorname{Ker}\left(\mathcal{O}^{\oplus r_{\ell}} \rightarrow \mathcal{O}^{\oplus r_{\ell-1}}\right)$ is (locally) free for large $\ell$, so we can replace such a module $\mathcal{O}^{\oplus r_{\ell}}$ with this kernel and 0 before that. Therefore Theorem 3.1 holds and we have

PROpOSITION 3.3. - Let $\mathcal{J}$ be a coherent subsheaf of $\mathcal{O}^{\oplus r_{0}}$ in a Stein manifold $X$. For each compact subset $K \subset X$ there is a residue current $R$ defined in a neighborhood of $K$ such that $\operatorname{ann} R=\mathcal{J}$.

The degree of explicitness of the Noetherian residue current $R$ in Theorem 1.1 is of course directly depending on the degree of explicitness of the resolution.

Example 1 (The Koszul complex). - Let $H$ be a Hermitian bundle over $X$ of rank $m$ and let $h$ be a non-trivial holomorphic section of the dual bundle $H^{*}$. Then $h$ can be considered as a morphism $H \rightarrow \mathbb{C} \times X$, and we get a generically exact complex (1.2) by taking $E_{k}=\Lambda^{k} H$ and let all the mappings $f_{k}$ be interior multiplication with $f$. If $\eta$ is the section of $E$ over $X \backslash Z$ of minimal norm such that $f \cdot \eta=1$, then $\sigma_{k} \xi=\eta \wedge \xi$ for sections $\xi$ of $E_{k-1}$, and hence $u_{k}^{\ell}=\eta \wedge(\bar{\partial} \eta)^{k-\ell-1}$, acting on $\Lambda^{\ell} H$ via wedge multiplication. Thus $R_{k}^{\ell}=\bar{\partial}|h|^{2 \lambda} \wedge \xi \wedge$ $\left.(\bar{\partial} \xi)^{k-\ell-1}\right|_{\lambda=0}$ are precisely the currents considered in [1]. If $h$ is a complete intersection and $h=h_{1} e_{1}^{*}+\cdots+h_{m} e_{m}^{*}$ in some local holomorphic frame $e_{j}^{*}$ for $H^{*}$, then $R$ is precisely the Coleff-Herrera product (1.1) times $e_{1} \wedge \cdots \wedge e_{m}$, where $e_{j}$ is the dual frame, see [1].

We now consider a simple example of a non-complete intersection ideal.

Example 2. - Consider the ideal $J=\left(z_{1}^{2}, z_{1} z_{2}\right)$ in $\mathbb{C}^{2}$ with zero variety $\left\{z_{1}=0\right\}$. It is easy to see that

$$
0 \rightarrow \mathcal{O} \stackrel{f_{2}}{\longrightarrow} \mathcal{O}^{\oplus 2} \stackrel{f_{1}}{\longrightarrow} \mathcal{O}
$$

where

$$
f_{1}=\left[\begin{array}{ll}
z_{1}^{2} & z_{1} z_{2}
\end{array}\right] \text { and } f_{2}=\left[\begin{array}{c}
z_{2} \\
-z_{1}
\end{array}\right]
$$

is a (minimal) resolution of $\mathcal{O} / J$. We equip the corresponding vector bundles with the trivial Hermitian metrics. Since $Z$ has codimension $1, R$ consists of the two parts $R_{2}=\left.\bar{\partial}|F|^{2 \lambda} \wedge u_{2}^{0}\right|_{\lambda=0}$ and $R_{1}=\left.\bar{\partial}|F|^{2 \lambda} \wedge u_{1}^{0}\right|_{\lambda=0}$, where $u_{2}^{0}=\sigma_{2} \bar{\partial} \sigma_{1}$ and $u_{1}^{0}=\sigma_{1}$, respectively. To compute $R$ it is enough to make a simple blow-up at the origin, and one gets, cf. [37] and [36], that

$$
R_{2}=\bar{\partial}\left[\frac{1}{z_{1}^{2}}\right] \wedge \bar{\partial}\left[\frac{1}{z_{2}}\right] \quad \text { and } \quad R_{1}=\left[\begin{array}{l}
0 \\
1
\end{array}\right]\left[\frac{1}{z_{2}}\right] \bar{\partial}\left[\frac{1}{z_{1}}\right]
$$


We see that ann $R_{2}=\left(z_{1}^{2}, z_{2}\right)$ and $\operatorname{ann} R_{1}=\left(z_{1}\right)$, and hence $\operatorname{ann} R=\left(z_{1}^{2}, z_{2}\right) \cap\left(z_{1}\right)=J$ as expected. Notice that the Koszul complex associated with the ideal $J$ is like (3.3) but with an extra factor $z_{1}$ in the mapping $f_{2}$. Then the current $R_{1}^{0}$ is of course the same as before, but

$$
R_{2}^{0}=\frac{1}{2} \bar{\partial}\left[\frac{1}{z_{1}^{3}}\right] \wedge \bar{\partial}\left[\frac{1}{z_{2}}\right]
$$

In this case ann $R^{0}=\operatorname{ann} R_{2}^{0} \cap \operatorname{ann} R_{1}^{0}=\left(z_{1}^{3}, z_{2}\right) \cap\left(z_{1}\right)$ which is strictly smaller than $J$. Roughly speaking, the annihilator of $R_{2}^{0}$ is too small, since the singularity of $\sigma_{2}$ and hence of $u_{2}^{0}$ is too big, due to the extra factor $z_{1}$ in $f_{2}$.

There has recently been a lot of work done on finding free resolutions of monomial ideals, see for example [28], [7] or [9]. For more involved explicit computations of residue currents for monomial ideals, see [37]. We conclude with a simple example where $\operatorname{ann}\left(\mathcal{O}\left(E_{0}\right) / J\right)=0$.

Example 3. - Consider the submodule $J$ of $\mathcal{O}^{\oplus 2}$ generated by $f_{1}=\left[z_{1} z_{2}-z_{1}^{2}\right]^{T}$ and the resolution $0 \rightarrow \mathcal{O} \stackrel{f_{1}}{\longrightarrow} \mathcal{O}^{\oplus 2}$, which is easily seen to be minimal. Notice that $Z=\left\{z_{1}=0\right\}$ is the associated set where $\mathcal{O}^{\oplus 2} / J$ is not locally free, or equivalently where $f_{1}$ is not locally constant. Moreover, notice that $\operatorname{ann}\left(\mathcal{O}^{\oplus 2} / J\right)=0$. The associated residue current is

$$
R=R_{1}=\left[\frac{1}{z_{2}}\right] \bar{\partial}\left[\frac{1}{z_{1}}\right]\left[\begin{array}{ll}
0 & 1
\end{array}\right] .
$$

If we extend the complex with the mapping $f_{0}=\left[z_{1} z_{1}\right]$ the new complex is still exact outside $Z$. Observe that ann $R$ is generated by $z_{1}[11]^{T}$ and moreover that $\operatorname{Ker} f_{0}$ is generated by $\left[z_{2}-z_{1}\right]^{T}$. Thus Ker $f_{0} \cap \operatorname{ann} R=J$ as expected.

\section{Cohen-Macaulay ideals and modules}

Let $\mathcal{F}_{x}$ be a $\mathcal{O}_{x}^{r}$-module. The minimal length $\nu_{x}$ of a resolution of $\mathcal{F}_{x}$ is precisely $n-\operatorname{depth} \mathcal{F}_{x}$, and $\operatorname{depth} \mathcal{F}_{x} \leqslant \operatorname{dim} \mathcal{F}_{x}$, so the length of the resolution is at least equal to $\operatorname{codim} \mathcal{F}_{x}$. Recall that the module $\mathcal{F}_{x}$ is Cohen-Macaulay if $\operatorname{depth} \mathcal{F}_{x}=\operatorname{dim} \mathcal{F}_{x}$, or equivalently, $\nu_{x}=\operatorname{codim} \mathcal{F}_{x}$, see [21]. As usual we say that an ideal $J_{x} \subset \mathcal{O}_{x}$ is CohenMacaulay if $\mathcal{F}_{x}=\mathcal{O}_{x} / J_{x}$ is a Cohen-Macaulay module.

A coherent analytic sheaf $\mathcal{F}$ is Cohen-Macaulay if $\mathcal{F}_{x}$ is Cohen-Macaulay for each $x$. If we have any locally free resolution of $\mathcal{F}$ and $\operatorname{codim} \mathcal{F}=p$, then at each point $\operatorname{Ker}\left(\mathcal{O}\left(E_{p-1}\right) \rightarrow\right.$ $\left.\mathcal{O}\left(E_{p-2}\right)\right)$ is free by the uniqueness theorem, see below, so by Oka's lemma the kernel is locally free; hence we can modify the given resolution to a locally free resolution of minimal length $p$. Notice that the residue current associated with a resolution of minimal length $p$ just consists of the single term $R=R_{p}^{0}$, which locally is a $r_{p} \times r_{0}$-matrix of currents.

THEOREM 4.1. - Suppose that $\mathcal{F}$ is a coherent analytic sheaf with codimension $p>0$ that is Cohen-Macaulay, and assume that

$$
0 \rightarrow \mathcal{O}\left(E_{p}\right) \rightarrow \cdots \rightarrow \mathcal{O}\left(E_{1}\right) \rightarrow \mathcal{O}\left(E_{0}\right)
$$

is a locally free resolution of $\mathcal{F}$ of minimal length $p$. Then the associated Noetherian current is independent of the Hermitian metric.

$4^{\text {e }}$ SÉRIE - TOME $40-2007-\mathrm{N}^{\circ} 6$ 
Proof. - Assume that $u$ and $u^{\prime}$ are the forms in $X \backslash Z$ constructed by means of two different choices of metrics on $E$. Then $\nabla_{\text {End }} u=I$ and $\nabla_{\text {End }} u^{\prime}=I$ in $X \backslash Z$, and hence

$$
\nabla_{\text {End }}\left(u u^{\prime}\right)=\left(\nabla_{\text {End }} u\right) u^{\prime}-u \nabla_{\text {End }} u^{\prime}=u^{\prime}-u,
$$

where the minus sign occurs since $u$ has odd order. For large $\operatorname{Re} \lambda$ we thus have, cf. the proof of Proposition 2.2,

$$
\nabla_{\text {End }}\left(|F|^{2 \lambda} u u^{\prime}\right)=|F|^{2 \lambda} u^{\prime}-|F|^{2 \lambda} u-\bar{\partial}|F|^{2 \lambda} \wedge u u^{\prime}
$$

As before one can verify that each term admits an analytic continuation to $\operatorname{Re} \lambda>-\epsilon$, and evaluating at $\lambda=0$ we get $\nabla_{\text {End }} W=U^{\prime}-U-M$, where $W=\left.|F|^{2 \lambda} u u^{\prime}\right|_{\lambda=0}$, and $M$ is the residue current

$$
M=\left.\bar{\partial}|F|^{2 \lambda} \wedge u u^{\prime}\right|_{\lambda=0} .
$$

Since $\nabla_{\text {End }}^{2}=0$, by Proposition 2.2 we therefore get

$$
R-R^{\prime}=\nabla_{\text {End }} M
$$

However, since the complex ends up at $p$, each term in $u u^{\prime}$ has at most bidegree $(0, p-2)$ and hence the current $M$ has at most bidegree $(0, p-1)$. Since it is supported on $Z$ with codimension $p$, it must vanish, cf. the proof of Proposition 2.2.

When $\mathcal{F}=\mathcal{O}\left(E_{0}\right) / \mathcal{J}$ is Cohen-Macaulay we can also define a cohomological residue that characterizes the module sheaf $\mathcal{J}=\operatorname{Im}\left(\mathcal{O}\left(E_{1}\right) \rightarrow \mathcal{O}\left(E_{0}\right)\right)$ locally. Suppose that we have a fixed resolution (4.1) of minimal length and let us assume that $p>1$. If $u$ is any solution to $\nabla_{\text {End }} u=I$ in $X \backslash Z$, then $u_{p}^{0}$ is a $\bar{\partial}$-closed $\operatorname{Hom}\left(E_{0}, E_{p}\right)$-valued $(0, p-1)$-form. Moreover if $u^{\prime}$ is another solution, then it follows from the preceding proof that $\bar{\partial}\left(u u^{\prime}\right)_{p}^{0}=u_{p}^{0}-u_{p}^{\prime 0}$. Therefore $u_{p}^{0}$ defines a Dolbeault cohomology class $\omega \in H^{0, p-1}\left(X \backslash Z, \operatorname{Hom}\left(E_{0}, E_{p}\right)\right)$. If $\phi$ is a holomorphic section of $E_{0}$ then $\omega \phi=\left[u_{p}^{0} \phi\right]$ is an element in $H^{0, p-1}\left(X \backslash Z, E_{p}\right)$. Moreover, if $v$ is any solution in $X \backslash Z$ to $\nabla v=\phi$, then $v_{p}$ defines the class $\omega \phi$. In fact, $\nabla(u v)=v-u \phi=v-u^{0} \phi$ so that $\bar{\partial}(u v)_{p}=u_{p}^{0} \phi-v_{p}$. Precisely as for a complete intersection, [18] and [31], we have the following cohomological duality principle.

THEOREM 4.2. - Let $X$ be a Stein manifold and let (4.1) be a resolution of minimal length $p$ of the Cohen-Macaulay sheaf $\mathcal{O}\left(E_{0}\right) / \mathcal{J}$ over $X$, and assume that $p>1$. Moreover, let $\omega$ be the associated class in $H^{0, p-1}\left(X \backslash Z, \operatorname{Hom}\left(E_{0}, E_{p}\right)\right)$. For a holomorphic section $\phi$ of $E_{0}$ the following conditions are equivalent:

(i) $\phi$ is a global section of $\mathcal{J}$.

(ii) The class $\omega \phi$ in $X \backslash Z$ vanishes.

(iii) $\int \omega \phi \wedge \bar{\partial} \xi=0$ for all $\xi \in \mathcal{D}_{n, n-p}\left(X, E_{p}^{*}\right)$ such that $\bar{\partial} \xi=0$ in a neighborhood of $Z$.

Notice that if $R$ is the associated Noetherian current, then $\bar{\partial} U_{p}^{0}=R_{p}$, so by Stokes' theorem, (iii) is equivalent to that $\int R_{p} \phi \wedge \xi=0$ for all $\xi \in \mathcal{D}_{n, n-p}\left(X, E_{p}^{*}\right)$ such that $\bar{\partial} \xi=0$ in a neighborhood of $Z$.

If $p=1$, then $f_{1}$ is an isomorphism outside $Z$, so its inverse $\omega=\sigma_{1}$ is a holomorphic $(0,0)$ form in $X \backslash Z$. Thus a holomorphic section $\phi$ of $E_{0}$ belongs to $\mathcal{J}$ if and only if $\omega \phi$ has a holomorphic extension across $Z$.

Proof. - If (i) holds, then $\phi=f_{1} \psi$ for some holomorphic $\psi$; thus $\nabla \psi=\phi$. However, since $p>1, \psi$ has no component in $E_{p}$, and hence by definition the class $\omega \phi$ vanishes. The implication (ii) $\rightarrow$ (iii) follows from Stokes' theorem. 
Let us now assume that (iii) holds, and choose a point $x$ on $Z$. Let $v_{k}=u_{k}^{0} \phi$. If $X^{\prime}$ is an appropriate small neighborhood of $x$, then, since $Z$ has codimension $p$ and $v_{p}$ is a $\bar{\partial}$-closed $(0, p)$-current, one can verify that the condition (iii) ensures that $\bar{\partial} w_{p}=v_{p}$ has a solution in $X^{\prime} \backslash \bar{W}$, where $W$ is a small neighborhood of $Z$ in $X^{\prime}$. Then, successively, all the lower degree equations $\bar{\partial} w_{k}=v_{k}+f_{k+1} w_{k+1}, k \geqslant 2$, can be solved in similar domains. Finally, we get a holomorphic solution $\psi=v_{1}+f_{2} w_{2}$ to $f_{1} \psi=\phi$, in such a domain. By Hartogs' theorem $\psi$ extends across $Z$ in $X^{\prime}$. Alternatively, one can obtain such a local holomorphic solution $\psi$, using the decomposition formula (5.4) below and mimicking the proof of the corresponding statement for a complete intersection in [31]; cf. also the proof of Proposition 7.1 in [5]. Since $X$ is Stein, one can piece together to a global holomorphic solution to $f_{1} \psi=\phi$, and hence $\phi$ is a section of $\mathcal{J}$.

Example 4. - Let $J$ be an ideal in $\mathcal{O}_{0}$ of dimension zero. Then it is Cohen-Macaulay and for each germ $\phi$ in $\mathcal{O}_{0}, \omega \phi$ defines a functional on $\mathcal{O}_{0}\left(E_{n}^{*}\right) \simeq \mathcal{O}_{0}^{r_{n}}$. If $J$ is defined by a complete intersection, then we may assume that (4.1) is the Koszul complex. Then $r_{n}=1$, and in view of the Dolbeault isomorphism, see, e.g., Proposition 3.2.1 in [31], $\omega \phi$ is just the classical Grothendieck residue.

For the rest of this section we will restrict our attention to modules over the local ring $\mathcal{O}_{0}$, and we let $\mathcal{O}\left(E_{k}\right)$ denote the free $\mathcal{O}_{0}$-module of germs of holomorphic sections at 0 of the vector bundle $E_{k}$. Given a free resolution (1.3) of a module $\mathcal{F}_{0}$ over $\mathcal{O}_{0}$ and given metrics on $E_{k}$ we thus get a germ $R$ of a Noetherian residue current at 0 . Recall that the resolution (1.3) is minimal if for each $k, f_{k}$ maps a basis of $\mathcal{O}\left(E_{k}\right)$ to a minimal set of generators of $\operatorname{Im} f_{k}$. The uniqueness theorem, see, e.g., Theorem 20.2 in [21], states that any two minimal (free) resolutions are equivalent, and moreover, that any (free) resolution has a minimal resolution as a direct summand.

For a Cohen-Macaulay module $\mathcal{F}_{0}$ over $\mathcal{O}_{0}$ we have the following uniqueness.

Proposition 4.3. - Let $\mathcal{F}_{0}$ be a Cohen-Macaulay module over $\mathcal{O}_{0}$ of codimension $p$. If we have two minimal free resolutions $\mathcal{O}\left(E_{\bullet}\right)$ and $\mathcal{O}\left(E_{\bullet}^{\prime}\right)$ of $\mathcal{F}_{0}$, then there are holomorphic invertible matrices $g_{p}$ and $g_{0}$ (local holomorphic isomorphism $g_{p}: E_{p}^{\prime} \simeq E_{p}$ and $g_{0}: E_{0}^{\prime} \simeq E_{0}$ ) such that $R=g_{p} R^{\prime} g_{0}^{-1}$.

Since minimal resolutions have minimal length $p$, the currents are independent of the metrics, in view of Proposition 4.1.

Proof. - By the uniqueness theorem there are holomorphic local isomorphisms $g_{k}: E_{k}^{\prime} \rightarrow E_{k}$ such that

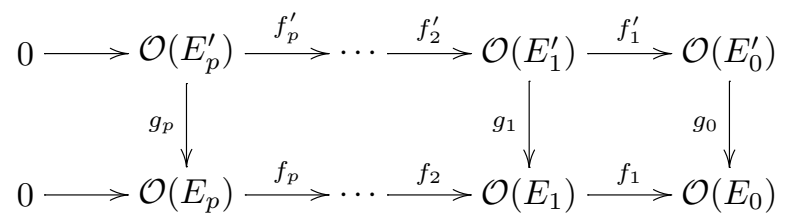

commutes. Let $g$ denote the induced isomorphism $E \rightarrow E^{\prime}$. Choose any metric on $E$ and equip $E^{\prime}$ with the induced metric, i.e., such that $|\xi|=\left|g^{-1} \xi\right|$ for a section $\xi$ of $E^{\prime}$. If $\sigma: E \rightarrow E$ and $\sigma^{\prime}: E^{\prime} \rightarrow E^{\prime}$ are the associated endomorphisms over $X \backslash Z$, cf. Section 2, then $\sigma^{\prime}=g \sigma g^{-1}$ in $X \backslash Z$, and therefore

$$
u^{\prime}=\sigma^{\prime}+\left(\bar{\partial} \sigma^{\prime}\right) \sigma^{\prime}+\cdots=g(\sigma+(\bar{\partial} \sigma) \sigma+\cdots) g^{-1}=g u g^{-1} .
$$

Therefore, $\left(u^{\prime}\right)_{p}^{0}=g_{p} u_{p}^{0} g_{0}^{-1}$, and hence the statement follows since $R=R_{p}=R_{p}^{0}$.

$4^{e}$ SÉRIE - TOME $40-2007-\mathrm{N}^{\circ} 6$ 
We shall now consider the residue current associated to a general free resolution.

THEOREM 4.4. - Let $\mathcal{F}_{0}$ be a Cohen-Macaulay module over $\mathcal{O}_{0}$ of codimension $p$. If $R$ is the residue current associated to an arbitrary free resolution (1.3) (and given metrics on $E_{k}$ ) and $R^{\prime}=R_{p}^{\prime}$ is associated to a minimal resolution $0 \rightarrow \mathcal{O}\left(E_{p}^{\prime}\right) \stackrel{f_{p}^{\prime}}{\longrightarrow} \cdots \stackrel{f_{2}^{\prime}}{\longrightarrow} \mathcal{O}\left(E_{1}^{\prime}\right) \stackrel{f_{1}^{\prime}}{\longrightarrow} \mathcal{O}\left(E_{0}^{\prime}\right)$, then

$$
R_{p}=h_{p} R_{p}^{\prime} \beta_{0},
$$

where $\beta_{0}: E_{0} \rightarrow E_{0}^{\prime}$ is a local holomorphic pointwise surjective morphism and $h_{p}$ is a local smooth pointwise injective morphism $h_{p}: E_{p}^{\prime} \rightarrow E_{p}$. Moreover, for each $\ell>0$,

$$
R_{p+\ell}=\alpha_{\ell} R_{p}
$$

where $\alpha_{\ell}$ is a smooth $\operatorname{Hom}\left(E_{p}, E_{p+\ell}\right)$-valued $(0, \ell)$-form.

Proof. - By the uniqueness theorem for resolutions, the resolution $E_{\bullet}^{\prime}$ is isomorphic to a direct summand in $E_{\bullet}$, and in view of the preceding proposition, we may assume that

$$
\mathcal{O}\left(E_{k}\right)=\mathcal{O}\left(E_{k}^{\prime} \oplus E_{k}^{\prime \prime}\right)=\mathcal{O}\left(E_{k}^{\prime}\right) \oplus \mathcal{O}\left(E_{k}^{\prime \prime}\right)
$$

and $f_{k}=f_{k}^{\prime} \oplus f_{k}^{\prime \prime}$, so that

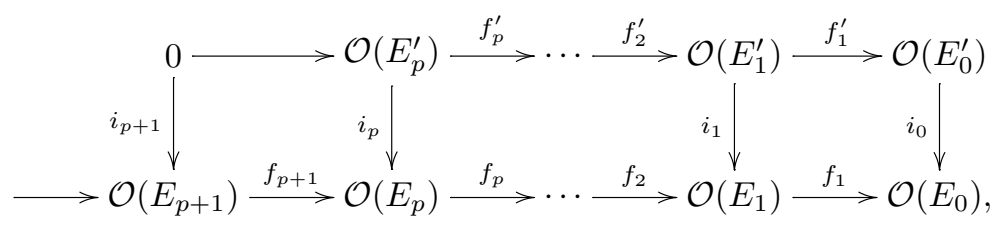

where $i_{k}: E_{k}^{\prime} \rightarrow E_{k}^{\prime} \oplus E_{k}^{\prime \prime}$ are the natural injections, and

$$
\rightarrow \mathcal{O}\left(E_{p+1}^{\prime \prime}\right) \stackrel{f_{p+1}^{\prime \prime}}{\longrightarrow} \mathcal{O}\left(E_{p}^{\prime \prime}\right) \stackrel{f_{p}^{\prime \prime}}{\longrightarrow} \cdots \stackrel{f_{2}^{\prime \prime}}{\longrightarrow} \mathcal{O}\left(E_{1}^{\prime \prime}\right) \stackrel{f_{1}^{\prime \prime}}{\longrightarrow} \mathcal{O}\left(E_{0}^{\prime \prime}\right)
$$

is a resolution of 0 . In particular,

$$
\rightarrow E_{p+1} \stackrel{f_{p+1}^{\prime \prime}}{\longrightarrow} E_{p}^{\prime \prime} \stackrel{f_{p}^{\prime \prime}}{\longrightarrow} \cdots \stackrel{f_{2}^{\prime \prime}}{\longrightarrow} E_{1}^{\prime \prime} \stackrel{f_{1}^{\prime \prime}}{\longrightarrow} E_{0}^{\prime \prime} \rightarrow 0
$$

is a pointwise exact sequence of vector bundles, and therefore the set $Z_{k}$ where $\operatorname{rank} f_{k}$ is not optimal coincides with the set $Z_{k}^{\prime}$ where rank $f_{k}^{\prime}$ is not optimal. In particular, $Z_{k}=\emptyset$ for $k>p$. If we choose, to begin with, Hermitian metrics on $E_{k}$ that respect this direct sum, and let $\sigma_{k}, \sigma_{k}^{\prime}$, and $\sigma_{k}^{\prime \prime}$ be the corresponding minimal inverses, then $\sigma_{k}=\sigma_{k}^{\prime} \oplus \sigma_{k}^{\prime \prime}$ and hence

$$
u_{k}^{0}=\left(\bar{\partial} \sigma_{k}^{\prime} \oplus \bar{\partial} \sigma_{k}^{\prime \prime}\right)\left(\bar{\partial} \sigma_{k-1}^{\prime} \oplus \bar{\partial} \sigma_{k-1}^{\prime \prime}\right) \cdots\left(\bar{\partial} \sigma_{2}^{\prime} \oplus \bar{\partial} \sigma_{2}^{\prime \prime}\right)\left(\sigma_{1}^{\prime} \oplus \sigma_{1}^{\prime \prime}\right)=\left(u^{\prime}\right)_{k}^{0} \oplus\left(u^{\prime \prime}\right)_{k}^{0}
$$

for all $k$. However, $\left(u^{\prime \prime}\right)_{k}^{0}$ is smooth, and hence

$$
R_{p}=R_{p}^{\prime} \oplus 0, \quad R_{k}=0 \text { for } k \neq p .
$$

For this particular choice of metric thus (4.4) holds with $h_{p}$ as the natural injection $i_{p}: E_{p}^{\prime} \rightarrow E_{p}$ and $\beta_{0}$ as the natural projection. 
Without any risk of confusion we can therefore from now on let $R_{p}^{\prime}$ denote the residue current with respect to this particular metric on $E$, and moreover let $\sigma^{\prime}$ denote the minimal inverse of $f$ with respect to this metric, etc. We now choose other metrics on $E_{k}$ and let $R_{k}$ from now on denote the residue current associated with this new metric. Following the notation in the proof of Proposition 4.1 we again have (4.3), and for degree reasons still $M_{p}^{0}=0$; here $M_{k}^{\ell}$ denotes the component of $M$ that takes values in $\operatorname{Hom}\left(E_{\ell}, E_{k}\right)$. Thus

$$
R_{p}-R_{p}^{\prime}=f_{p+1} M_{p+1}^{0}
$$

Moreover, if we expand $u u^{\prime}$, we get

$$
\begin{aligned}
M_{p+1}^{0}= & \bar{\partial}|F|^{2 \lambda} \wedge\left[\sigma_{p+1} \sigma_{p}^{\prime}\left(\bar{\partial} \sigma_{p-1}^{\prime}\right) \cdots\left(\bar{\partial} \sigma_{1}^{\prime}\right)\right. \\
& \left.+\sigma_{p+1}\left(\bar{\partial} \sigma_{p}\right) \sigma_{p-1}^{\prime}\left(\bar{\partial} \sigma_{p-2}^{\prime}\right) \cdots\left(\bar{\partial} \sigma_{1}^{\prime}\right)+\cdots\right]\left.\right|_{\lambda=0} .
\end{aligned}
$$

However, $\sigma_{p+1}\left(\bar{\partial} \sigma_{p}\right)=\left(\bar{\partial} \sigma_{p+1}\right) \sigma_{p}$ and $\sigma_{p+1}$ is smooth since $Z_{p+1}$ is empty, so

$$
M_{p+1}^{0}=-\sigma_{p+1} R_{p}^{\prime}+\left(\bar{\partial} \sigma_{p+1}\right) M_{p}^{0}=-\sigma_{p+1} R_{p}^{\prime} .
$$

Thus,

$$
R_{p}=R_{p}^{\prime}-f_{p+1} \sigma_{p+1} R_{p}^{\prime}=\left(I_{E_{p}}-f_{p+1} \sigma_{p+1}\right) R_{p}^{\prime} .
$$

Since $f_{p+1}$ has constant rank, $H=\operatorname{Im} f_{p+1}$ is a smooth subbundle of $E_{p}$. Notice that $\Pi=$ $I_{E_{p}}-f_{p+1} \sigma_{p+1}$ is the orthogonal projection of $E_{p}$ onto the orthogonal complement of $H$ with respect to the new metric. In this case therefore $h$ in (4.4) becomes the natural injection $i_{p}: E_{p}^{\prime} \rightarrow E_{p}$ composed by $\Pi$, and since $E_{p}^{\prime} \cap H=0, h$ is pointwise injective.

Since $Z_{k}$ is empty for $k>p, \sigma_{k}$ is smooth for $k>p$ and hence for $\ell>p$,

$$
R_{\ell}=\bar{\partial}|F|^{2 \lambda} \wedge\left(\bar{\partial} \sigma_{\ell}\right) \cdots\left(\bar{\partial} \sigma_{p+1}\right) u_{p}^{0}=\left(\bar{\partial} \sigma_{\ell}\right) \cdots\left(\bar{\partial} \sigma_{p+1}\right) \bar{\partial}|F|^{2 \lambda} \wedge u_{p}^{0}=\alpha_{\ell} R_{p}
$$

where $\alpha_{\ell}=\left(\bar{\partial} \sigma_{\ell}\right) \cdots\left(\bar{\partial} \sigma_{p+1}\right)$.

\section{Division and interpolation formulas}

To obtain formulas for division and interpolation that involve our currents $R$ and $U$ we will use the general scheme developed in [5]. Let $z$ be a fixed point in $\mathbb{C}^{n}$, let $\delta_{\zeta-z}$ denote interior multiplication by the vector field $2 \pi i \sum_{1}^{n}\left(\zeta_{j}-z_{j}\right)\left(\partial / \partial \zeta_{j}\right)$, and let $\nabla_{\zeta-z}=\delta_{\zeta-z}-\bar{\partial}$. Let $g=g_{0,0}+\cdots+g_{n, n}$ be a smooth form such that $\nabla_{\zeta-z} g=0$ and $g_{0,0}(z)=1$ (here lower indices denote bidegree); such a form will be called a weight with respect to the point $z$. If $g$ has compact support then

$$
\phi(z)=\int g \phi
$$

for $\phi$ that are holomorphic in a neighborhood of the support of $g$, [5].

Let $D$ be a ball with center at the origin in $\mathbb{C}^{n}$ and let $\chi$ be a cutoff function that is 1 in a neighborhood of $\bar{D}$. Then for each $z \in \bar{D}$,

$$
g=\chi-\bar{\partial} \chi \wedge \frac{s}{\nabla_{\zeta-z} s}=\chi-\bar{\partial} \chi \wedge\left[s+s \wedge \bar{\partial} s+\cdots+s \wedge(\bar{\partial} s)^{n-1}\right]
$$

$4^{\mathrm{e}}$ SÉRIE - TOME $40-2007-\mathrm{N}^{\circ} 6$ 
is a weight, and it depends holomorphically on $z$. Assume that (2.1) is a complex of (trivial) bundles over a neighborhood of $\bar{D}$ and let $\mathcal{J}=\operatorname{Im} f_{1}$. Let us also fix global frames for the bundles $E_{k}$. Then $E_{k} \simeq \mathbb{C}^{\operatorname{rank} E_{k}}$ and the morphisms $f_{k}$ are just matrices of holomorphic functions. One can find (see [5] for explicit choices) $(k-\ell, 0)$-form-valued holomorphic Hefer morphisms, i.e., matrices, $H_{k}^{\ell}: E_{k} \rightarrow E_{\ell}$ depending holomorphically on $z$ and $\zeta$, such that $H_{k}^{\ell}=0$ for $k<\ell, H_{\ell}^{\ell}=I_{E_{\ell}}$, and in general,

$$
\delta_{\zeta-z} H_{k}^{\ell}=H_{k-1}^{\ell} f_{k}-f_{\ell+1}(z) H_{k}^{\ell+1} ;
$$

here $f$ stands for $f(\zeta)$. Let

$$
H U=\sum_{\ell} H^{\ell+1} U=\sum_{\ell k} H_{k}^{\ell+1} U_{k}^{\ell}, \quad H R=\sum_{\ell} H^{\ell} R=\sum_{\ell k} H_{k}^{\ell} R_{k}^{\ell}
$$

Then $g^{\prime}=f(z) H U+H U f+H R$ maps a section of $E_{\ell}$ depending on $\zeta$ into a (current-valued) section of $E_{\ell}$ depending on both $\zeta$ and $z$. Moreover, $\nabla_{\zeta-z} g^{\prime}=0$ and $g_{0,0}^{\prime}=I_{E}$. If $g$ is weight with compact support, cf. Proposition 5.4 in [5], we therefore have the representation

$$
\phi(z)=f_{k+1}(z) \int_{\zeta} H^{k+1} U \phi \wedge g+\int_{\zeta} H^{k} U f_{k} \phi \wedge g+\int_{\zeta} H^{k} R \phi \wedge g,
$$

$z \in \bar{D}$, for $\phi \in \mathcal{O}\left(\bar{D}, E_{k}\right)$. Thus we get an explicit realization (in terms of $U$ ) of $f_{k+1} \psi=\phi$, if $f_{k} \phi=0$ and $R \phi=0$, and thus an explicit proof of Proposition 2.3(i).

If we have a complex (1.2) over a neighborhood of $\bar{D}$, and either $f_{1}$ is generically surjective or we have an extension to a generically exact complex ending at $E_{-1}$, then (5.4) still holds for $k=0$. If $R$ is Noetherian, then the last two terms vanish if and only if $\phi$ is in $\mathcal{J}$. We thus obtain an explicit realization of the membership of $\mathcal{J}$.

In the same way as in [2] one can extend these formulas slightly, to obtain a characterization of the module $\mathcal{E} \mathcal{J}$ of smooth tuples of functions generated by $\mathcal{J}$, i.e., the set of all $\phi=f_{1} \psi$ for smooth $\psi$. For simplicity we assume that $\mathcal{O}\left(E_{0}\right) / \mathcal{J}$ has positive codimension so that $f_{0}=0$. Let $R$ be a Noetherian current for $J$. First notice that if $\phi=f_{1} \psi$, then, cf. Proposition 2.2, $R \phi=R^{0} \phi=R^{0} f_{1} \psi-R^{1} \bar{\partial} \psi=R \nabla \psi=\nabla R^{1} \psi=0$, so that $R \phi=0$. Since each partial derivative $\partial / \partial \bar{z}_{j}$ commutes with $f_{1}$, we get that

$$
R\left(\partial^{\alpha} \phi / \partial \bar{z}^{\alpha}\right)=0
$$

for all multiindices $\alpha$. The converse can be proved by integral formulas precisely as in [2], and thus we have

THEOREM 5.1. - Assume that $\mathcal{J} \subset \mathcal{O}^{\oplus r_{0}}$ is a coherent subsheaf such that $\mathcal{O}^{\oplus r_{0}} / \mathcal{J}$ has positive codimension, and let $R$ be a Noetherian residue current for $\mathcal{J}$. Then an $r_{0}$-tuple $\phi \in \mathcal{E}^{\oplus r_{0}}$ of smooth functions is in $\mathcal{E} \mathcal{J}$ if and only if (5.5) holds for all $\alpha$.

Let $\mathcal{J}$ be a coherent Cohen-Macaulay ideal sheaf of codimension $p$ over some pseudoconvex set $X$ and let $\mu$ be an analytic functional that annihilates $\mathcal{J}$. In [19] was proved (Theorem 4.4) that $\mu$ can be represented by an $(n, n)$-current $\tilde{\mu}$ with compact support of the form $\tilde{\mu}=\alpha \wedge R$, where $\alpha$ is a smooth $(n, n-p)$-form with compact support and $R$ is the Coleff-Herrera product of a complete intersection ideal contained in $\mathcal{J}$. In particular, $\tilde{\mu}$ vanishes on $\mathcal{E} \mathcal{J}$. As another application of our integral formulas we prove the following more general result. 
THEOREM 5.2. - Let $X$ be a pseudoconvex set in $\mathbb{C}^{n}$ and let $\mathcal{J}$ be a coherent subsheaf of $\mathcal{O}\left(E_{0}\right) \simeq \mathcal{O}^{\oplus r_{0}}$ such that $\mathcal{O}\left(E_{0}\right) / \mathcal{J}$ has positive codimension. If $\mu \in \mathcal{O}^{\prime}\left(X, E_{0}^{*}\right)$ is an analytic functional that vanishes on $\mathcal{J}$, then there is an $(n, n)$-current $\tilde{\mu}$ with compact support that represents $\mu$, i.e.,

$$
\mu . \xi=\tilde{\mu} . \xi, \quad \xi \in \mathcal{O}\left(X, E_{0}\right),
$$

and such that $\tilde{\mu}$ vanishes on $\mathcal{E} J$. More precisely we can choose $\tilde{\mu}$ of the form

$$
\tilde{\mu}=\sum_{k} \alpha_{k} R_{k}
$$

where $R$ is a Noetherian residue current for $\mathcal{J}$ and $\alpha_{k} \in \mathcal{D}_{n, n-k}\left(X, E_{k}^{*}\right)$.

Here $E_{k}$ refers to the trivial vector bundles associated to a free resolution of $\mathcal{O}\left(E_{0}\right) / \mathcal{J}$.

Proof. - Assume that $\mu$ is carried by the $\mathcal{O}(X)$-convex compact subset $K \subset X$ and let $V$ be an open neighborhood of $K$. For each $z \in V$ we can choose a weight $g^{z}$ with respect to $z$, such that $z \mapsto g^{z}$ is holomorphic in $V$ and all $g^{z}$ have support in some compact $\tilde{K} \subset X$, see Example 10 in [1]. Let $R$ be a residue current for $\mathcal{J}$, associated to a free resolution of $\mathcal{O}\left(E_{0}\right) / \mathcal{J}$ in a neighborhood of $\tilde{K}$, cf. Proposition 3.3. Now consider the corresponding decomposition (5.4) (with $k=0$ ) that holds for $z \in V$, with $g=g^{z}$; notice that $f_{0}=0$ by the assumption on $\mathcal{J}$. The analytic functional $\mu$ has a continuous extension to $\mathcal{O}\left(K, E_{0}\right)$ and since $\mathcal{O}(X)$ is dense in $\mathcal{O}(K) \mu$ will vanish on the first term on the right-hand side in (5.4). If we define the $(n, n)$ current

$$
\tilde{\mu}=\mu_{z}\left(g^{z} \wedge H^{0}\right) R=\sum_{k} \mu_{z}\left(g_{n-k, n-k}^{z} \wedge H_{k}^{0}\right) R_{k}=\sum_{k} \alpha_{k} R_{k},
$$

then $\alpha_{k}$ have compact support and (5.6) holds. Since $R$ is Noetherian, $\tilde{\mu}$ annihilates $\mathcal{E} \mathcal{J}$.

\section{Homogeneous residue currents}

We will now make a construction of homogeneous Noetherian residue currents in $\mathbb{C}^{n+1}$. This is the key to find global Noetherian currents for polynomial ideals in $\mathbb{C}^{n}$ by homogenization in the next section. Let $S=\mathbb{C}\left[z_{0}, z_{1}, \ldots, z_{n}\right]$ be the graded ring of polynomials in $\mathbb{C}^{n+1}$, and let $S(-d)$ be equal to $S$ considered as an $S$-module, but with the grading shifted by $-d$, so that the constants have degree $d$, the linear forms have degree $d+1$, etc. Assume that

$$
0 \rightarrow M_{N} \rightarrow \cdots \rightarrow M_{1} \rightarrow M_{0}
$$

is a complex of free graded $S$-modules, where

$$
M_{0}=S^{\oplus r_{0}}, \quad M_{k}=S\left(-d_{1}^{k}\right) \oplus \cdots \oplus S\left(-d_{r_{k}}^{k}\right) .
$$

Then the (degree preserving) mappings are given by matrices of homogeneous elements in $S$. We can associate to (6.1) a generically exact complex of vector bundles (1.2) over $\mathbb{P}^{n}$ in the following way. Let $\mathcal{O}(\ell)$ be the holomorphic line bundle over $\mathbb{P}^{n}$ whose sections are (naturally identified with) $\ell$-homogeneous functions in $\mathbb{C}^{n+1}$. Moreover, let $E_{j}^{i}$ be disjoint trivial line bundles over $\mathbb{P}^{n}$ and let

$$
E_{k}=\left(E_{1}^{k} \otimes \mathcal{O}\left(-d_{1}^{k}\right)\right) \oplus \cdots \oplus\left(E_{r_{k}}^{k} \otimes \mathcal{O}\left(-d_{r_{k}}^{k}\right)\right)
$$

$4^{\text {e }}$ SÉRIE - TOME $40-2007-\mathrm{N}^{\circ} 6$ 
Notice that the homogeneous elements in $M_{\ell}$ of degree $r$ precisely correspond to the global holomorphic sections of the bundle $E_{\ell} \otimes \mathcal{O}(r)$.

The mappings in (6.1) induce vector bundle morphisms $f_{k}: E_{k} \rightarrow E_{k-1}$. We equip $E_{k}$ with the natural Hermitian metric, i.e., such that

$$
|\xi(z)|_{E_{k}}^{2}=\sum_{j=1}^{r_{k}}\left|\xi_{j}(z)\right|^{2}|z|^{2 d_{j}^{k}},
$$

if $\xi=\left(\xi_{1}, \ldots, \xi_{r_{k}}\right)$, and we have the associated currents $U$ and $R$ as before; they are associated to the complex

$$
0 \rightarrow E_{N} \otimes \mathcal{O}(r) \stackrel{f_{N}}{\longrightarrow} \cdots \stackrel{f_{2}}{\longrightarrow} E_{1} \otimes \mathcal{O}(r) \stackrel{f_{1}}{\longrightarrow} E_{0} \otimes \mathcal{O}(r)
$$

as well.

Example 5. - For each $j, k$ let $\epsilon_{j}^{k}$ be a global frame element for the bundle $E_{j}^{k}$. Then

$$
R_{k}^{\ell}=\sum_{i=1}^{r_{\ell}} \sum_{j=1}^{r_{k}}\left(R_{k}^{\ell}\right)_{i j} \otimes \epsilon_{i}^{k} \otimes\left(\epsilon_{j}^{\ell}\right)^{*},
$$

where each $\left(R_{k}^{\ell}\right)_{i j}$ is a $(0, k-\ell)$-current on $\mathbb{P}^{n}$, taking values in $\operatorname{Hom}\left(\mathcal{O}\left(-d_{j}^{\ell}\right), \mathcal{O}\left(-d_{i}^{k}\right)\right) \simeq$ $\mathcal{O}\left(d_{j}^{\ell}-d_{i}^{k}\right)$; alternatively $\left(R_{k}^{\ell}\right)_{i j}$ can be viewed as a $\left(d_{j}^{\ell}-d_{i}^{k}\right)$-homogeneous current on $\mathbb{C}^{n+1} \backslash\{0\}$. In the affine part $\mathcal{U}_{0}=\left\{[z] \in \mathbb{P}^{n} ; z_{0} \neq 0\right\}$ we have, for each $k$, a holomorphic frame

$$
e_{j}^{k}=z_{0}^{-d_{j}^{k}} \epsilon_{j}^{k}, \quad j=1, \ldots, r_{k},
$$

for the bundle $E_{k}$. In these frames

$$
R_{k}^{\ell}=\sum_{i=1}^{r_{\ell}} \sum_{j=1}^{r_{k}}\left(\hat{R}_{k}^{\ell}\right)_{i j} \otimes e_{i}^{k} \otimes\left(e_{j}^{\ell}\right)^{*},
$$

where $\left(\hat{R}_{k}^{\ell}\right)_{i j}$ are (scalar-valued) currents in $\mathcal{U}_{0} \simeq \mathbb{C}^{n}$. Since $\left(\hat{R}_{k}^{\ell}\right)_{i j}$ are the dehomogenizations of $\left(R_{k}^{\ell}\right)_{i j}$, and $d_{j}^{\ell}-d_{i}^{k} \leqslant 0$, it is easily seen that $\left(\hat{R}_{k}^{\ell}\right)_{i j}$ have current extensions to $\mathbb{P}^{n}$.

If (6.1) is exact, then according to the Buchsbaum-Eisenbud theorem for graded rings, see [22], the set in $\mathbb{C}^{n+1}$ (or equivalently in $\mathbb{P}^{n}$ ) where the rank of $f_{k}$ is strictly less than the generic rank $\rho_{k}$, has at least codimension $k$. It follows, cf. the proof of Theorem 3.1, that $R^{\ell}=0$ for $\ell \geqslant 1$, and (6.2) is exact. In particular, $R=R^{0}$ is a Noetherian residue current for the subsheaf $\mathcal{J} \otimes \mathcal{O}(r)$ of $\mathcal{O}\left(E_{0} \otimes \mathcal{O}(r)\right)$ generated by $f_{1}$. Let now $\phi$ be a global holomorphic section $\phi$ of $E_{0} \otimes \mathcal{O}(r)$, that is generically in the image of $f_{1}$, and such that $R \phi=0$. Then $\nabla\left(U^{0} \phi\right)=\phi$, cf. the proof of Proposition 2.3, and we obtain a holomorphic section $\psi$ of $E_{1} \otimes \mathcal{O}(r)$ such that $f_{1} \psi=\phi$, provided that we can solve globally a sequence of $\bar{\partial}$-equations. The first one is $\bar{\partial} w_{\mu}=U_{\mu}^{0} \phi, \mu=\min (N-1, n)$, and the right hand side here is a $(0, \mu-1)$-current with values in

$$
E_{\mu} \otimes \mathcal{O}(r) \simeq \bigoplus_{j=1}^{r_{\mu}} \mathcal{O}\left(r-d_{j}^{\mu}\right)
$$


Recall that $H^{0, q}\left(\mathbb{P}^{n}, \mathcal{O}(\nu)\right)=0$ for all $\nu$ if $0<q<n$, whereas $H^{0, n}\left(\mathbb{P}^{n}, \mathcal{O}(\nu)\right)=0$ if $\nu \geqslant-n$, see, e.g., [17]. Therefore the equation has a global solution if either $N \leqslant n$ or $\max _{j}\left(r-d_{j}^{n}\right) \geqslant-n$. The other equations to solve, $\bar{\partial} w_{k}=U_{k}^{0} \phi+f_{k+1} w_{k+1}$, have lower degree so then there are no cohomological obstructions. Thus we have:

Proposition 6.1. - Assume that $J \subset M_{0}$ is a homogeneous submodule and (6.1) a free resolution of $M_{0} / J$ of minimal length, and let $R$ be the associated Noetherian residue current. Let $\phi$ be a holomorphic section of $E_{0} \otimes \mathcal{O}(r)$ that lies generically in the image of $f_{1}: E_{1} \otimes$ $\mathcal{O}(r) \rightarrow E_{0} \otimes \mathcal{O}(r)$. If either

(i) $N \leqslant n$

or

(ii) $r \geqslant \max _{j}\left(d_{j}^{n+1}\right)-n$, then $f_{1} \psi=\phi$ has a global holomorphic solution if (and only if) $R \phi=0$.

Let (6.1) be any complex and let $R$ be the associated residue current. If we in addition assume that (6.1) has length at most $n+1$, then by a similar argument as above it follows that (6.1) is exact if and only if $R^{\ell}=0$ for all $\ell \geqslant 1$, i.e., if and only if (6.2) is exact, cf. Theorem 3.1.

Remark 2. - The minimal length $N$ of a resolution (6.1) is equal to $n+1-\operatorname{depth}\left(M_{0} / J\right)$ by the Auslander-Buchsbaum theorem, see [21]. The condition (i) is equivalent to that $\operatorname{depth}\left(M_{0} / J\right) \geqslant 1$ which means that $M_{0} / J$ contains a nontrivial nonzerodivisor. If $J$ is defined by a complete intersection, then the condition (i) is fulfilled. Also if $Z$ is discrete and all the zeros are of first order, then depth $S / J=1$, see [22], so that (i) holds.

The least possible value of $r$ in (ii) is closely related to the degree of regularity of $J$, see, e.g., [22]. An estimate of the regularity for zero-dimensional ideals is given in [35]. See [8] for a general criterion for a given degree of regularity. See also Remark 3 below.

\section{Noetherian residue currents for polynomial ideals}

We will now use the results from the previous section to obtain Noetherian residue currents for (sheaves induced by) polynomial modules in $\mathbb{C}^{n}$. Let $z^{\prime}=\left(z_{1}, \ldots, z_{n}\right)$ be the standard coordinates in $\mathbb{C}^{n}$ that we identify with $\mathcal{U}_{0}=\left\{[z] \in \mathbb{P}^{n} ; z_{0} \neq 0\right\}$, where $[z]=\left[z_{0}, \ldots, z_{n}\right]$ are the usual homogeneous coordinates on $\mathbb{P}^{n}$. Let $F_{1}$ be a $\operatorname{Hom}\left(\mathbb{C}^{r_{1}}, \mathbb{C}^{r_{0}}\right)$-valued polynomial in $\mathbb{C}^{n}$, whose columns $F^{1}, \ldots, F^{r_{1}}$ have (at most) degrees $d_{1}^{1}, \ldots, d_{r_{1}}^{1}$ and let $J$ be the submodule of $\mathbb{C}\left[z_{1}, \ldots, z_{n}\right]^{r_{0}}$ generated by $F^{1}, \ldots, F^{r_{1}}$. After the homogenizations $f^{k}(z)=z_{0}^{d_{k}^{1}} F^{k}\left(z^{\prime} / z_{0}\right)$ we get an $r_{0} \times r_{1}$-matrix $f_{1}$ whose columns are $d_{k}^{1}$-homogeneous forms in $\mathbb{C}^{n+1}$; thus a graded mapping

$$
f_{1}: S\left(-d_{1}^{1}\right) \oplus \cdots \oplus S\left(-d_{r_{1}}^{1}\right) \rightarrow S^{\oplus r_{0}} .
$$

Extending to a graded resolution (of minimal length) (6.1) we obtain a Noetherian residue current $R$ for the sheaf generated by $f_{1}$ and an associated current $U$. In the trivializations in $\mathbb{C}^{n} \simeq \mathcal{U}_{0}$, described in Example 5, the component $R_{k}$ of $R$ is the matrix $\left(\hat{R}_{k}^{0}\right)_{i j}$. In the same trivializations $U_{k}^{\ell}$ corresponds to a matrix $\left(\hat{U}_{k}^{\ell}\right)_{i j}$. Moreover, the mappings $f_{k}$ correspond to the matrices $F_{k}$ that are just the dehomogenizations of the matrices $f_{k}$ in (1.2).

If $\Phi$ is an $r_{0}$-tuple of polynomials in $\mathbb{C}^{n}$ and there is a tuple $\Psi$ of polynomials such that $\Phi=F_{1} \Psi$ in $\mathbb{C}^{n}$ then clearly $R \Phi=0$. Conversely, if $R \Phi=0$ in $\mathbb{C}^{n}$ (and the equation is locally solvable generically) we know that $\Phi$ is in the sheaf generated by $F_{1}$ and hence by Cartan's theorem there is a polynomial solution to $F_{1} \Psi=\Phi$. However, we now have a procedure to find such a $\Psi$ : Take a homogenization $\phi(z)=z_{0}^{r} \Phi\left(z^{\prime} / z_{0}\right)$ for some $r \geqslant \operatorname{deg} \Phi$. The condition

$4^{\text {e }}$ SÉRIE - TOME $40-2007-\mathrm{N}^{\circ} 6$ 
$R \Phi=0$ in $\mathbb{C}^{n}$ means that $R \phi=0$ outside the hyperplane at infinity, so if $r$ is large enough, $R \phi=0$ on $\mathbb{P}^{n}$. Now Proposition 6.1 applies if either $r$ is so large that condition (ii) is fulfilled, or if the length of the resolution is less than $n+1$. If $r$ is chosen large enough we thus have a holomorphic section $\psi$ of $E_{1} \otimes \mathcal{O}(r)$ such that $f_{1} \psi=\phi$. After dehomogenization we get the desired polynomial solution $\Psi=\left(\Psi^{j}\right)$ to $F_{1} \Psi=\sum F^{j} \Psi^{j}=\Phi$, and $\operatorname{deg} F^{j} \Psi^{j} \leqslant r$. It is well known that in the worst case the final degree has to be doubly exponential; at least $d^{2^{(n / 10)}}$, if $d$ is the degree of $F_{1}$, see [27].

Remark 3. - The final degree is essentially depending on the maximal polynomial degree in the resolution, and it is known to be at worst like $(2 d)^{2^{n}-1}$ if $d$ is the degree of the generators, see [6].

We proceed with a result where we have optimal control of the degree of the solution; it is a generalization of Max Noether's classical theorem, [29]; see also [23].

THEOREM 7.1. - Let $F^{1}, \ldots, F^{r_{1}}$ be $r_{0}$-columns of polynomials in $\mathbb{C}^{n}$ and let $J$ be the homogeneous submodule of $M_{0}=S^{\oplus r_{0}}$ defined by the homogenized forms $f^{1}, \ldots, f^{r_{1}}$. Furthermore, assume that the quotient module $M_{0} / J$ is Cohen-Macaulay and that no irreducible component of $Z$ is contained in the hyperplane at infinity. If $\Phi$ belongs to the submodule $\tilde{J} \subset \mathbb{C}\left[z^{\prime}\right]^{r_{0}}$ generated by $F^{1}, \ldots, F^{r_{1}}$, then there are tuples of polynomials $\Psi^{j}$ with $\operatorname{deg}\left(F^{j} \Psi^{j}\right) \leqslant \operatorname{deg} \Phi$ such that $F^{1} \Psi^{1}+\cdots+F^{r_{1}} \Psi^{r_{1}}=\Phi$.

Sketch of proof. - We follow the procedure described above. Assume that codim $M_{0} / J=p$. The Cohen-Macaulay assumption means that $\operatorname{dim} M_{0} / J=\operatorname{depth} M_{0} / J=n+1-$ $\operatorname{codim} M_{0} / J$. By the Auslander-Buchsbaum theorem therefore we can choose a resolution (6.1) of $M_{0} / J$ of length $p$, see [22]. Moreover all irreducible components of $Z$ have codimension $p$. We choose $r=\operatorname{deg} \Phi$. Since $\Phi$ is in the ideal in $\mathbb{C}^{n}$ we have that $R \phi=0$ in $\mathbb{C}^{n}$. By Proposition 2.2, $R=R_{p}$ and since $Z$ has no component contained in the hyperplane at infinity, we can copy the argument in the proof of Theorem 1.2 in [3] and conclude that $R \phi=0$ in $\mathbb{P}^{n}$. Since $p<n+1$, cf. Proposition 6.1, we can find a holomorphic section $\psi$ of $E_{1} \otimes \mathcal{O}(r)$ such that $f_{1} \psi=\phi$. After dehomogenization we get the desired solution $\Psi$.

We conclude this section with an explicit integral formula that provides a realization of the membership of $\Phi$ in $J \subset \mathbb{C}\left[z_{1}, \ldots, z_{n}\right]^{r_{0}}$; for simplicity we assume that the matrix $F_{1}=$ $\left(F^{1}, \ldots, F^{r_{1}}\right)$ is generically surjective, i.e., has generic rank $r_{0}$. From now on we write $z$ rather than $z^{\prime}$. It is easy to see that one can choose Hefer matrices of forms $H_{k}^{\ell}$ satisfying (5.3) (with $f_{k}$ replaced by $F_{k}$ ) that are polynomials in both $z$ and $\zeta$; in fact, the explicit formula in Section 4 in [5] when applied to polynomials will produce polynomials. Notice that

$$
g=\frac{1+\langle\bar{\zeta}, z\rangle}{1+|\zeta|^{2}}+\frac{i}{2 \pi} \partial \bar{\partial} \log \left(1+|\zeta|^{2}\right)
$$

is a weight in $\mathbb{C}^{n}$ with respect to the point $z$, cf. Section 5. Since $g^{\mu}=\mathcal{O}\left(1 /|\zeta|^{\mu}\right)$ for fixed $z$ and $H^{\ell}$ consists of polynomials, it follows that

$$
g^{\mu} \wedge H^{0} R, \quad g^{\mu} \wedge H^{1} U
$$

have current extensions to $\mathbb{P}^{n}$ if $\mu$ is large enough, cf. Example 5. Let $\chi_{k}(\zeta)=\chi(|\zeta| / k)$, where $\chi(t)$ is a cutoff function that is 1 for $t<1$ and 0 for $t>2$. If $\mu$ is sufficiently large, depending on the order at infinity of $R$ and $U$, we have that 


$$
\begin{gathered}
\chi_{k} g^{\mu} \wedge H^{0} R \rightarrow g^{\mu} \wedge H^{0} R, \quad \bar{\partial} \chi_{k} \wedge g^{\mu} \wedge H^{0} R \rightarrow 0, \\
\chi_{k} g^{\mu} \wedge H^{1} U \rightarrow g^{\mu} \wedge H^{1} U, \quad \bar{\partial} \chi_{k} \wedge g^{\mu} \wedge H^{1} U \rightarrow 0, \quad k \rightarrow \infty .
\end{gathered}
$$

Let $g_{k}=\chi_{k}-\bar{\partial} \chi_{k} \wedge s / \nabla_{\zeta-z} s$, where $s$ is the $(1,0)$-form in (5.2). Then $g_{k} \wedge g^{\mu+m}$ is a compactly supported weight with respect to $z$ if $k>|z|$, and hence we have the representation (writing $F$ rather than $F_{1}$ )

$$
\Phi(z)=F(z) \int g_{k} \wedge g^{\mu+m} \wedge H^{1} U \Phi+\int g_{k} \wedge g^{\mu+m} \wedge H^{0} R \Phi .
$$

Notice that

$$
\left(\frac{1+\langle\bar{\zeta}, z\rangle}{1+|\zeta|^{2}}\right)^{m} P(\zeta)
$$

is smooth on $\mathbb{P}^{n}$ for fixed $z$ if $P$ is a polynomial with $\operatorname{deg} P \leqslant m$. If we let $k \rightarrow \infty$ we therefore obtain

THEOREM 7.2. - Let $F$ be a $r_{0} \times r_{1}$-matrix of polynomials in $\mathbb{C}^{n}$ with generic rank $r_{0}$ and let $J$ be the submodule of $\mathbb{C}\left[z_{1}, \ldots, z_{n}\right]^{r_{0}}$ generated by the columns of $F$. For each given integer $m$, with the notation above and for a large enough $\mu$, we have the polynomial decomposition

$$
\Phi(z)=F(z) \int g^{\mu+m} \wedge H^{1} U \Phi+\int g^{\mu+m} \wedge H^{0} R \Phi
$$

of $r_{0}$-columns $\Phi$ of polynomials with degree at most $m$, and the last term vanishes as soon as $\Phi \in J$.

The integrals here are to be interpreted as the action of currents on test functions on $\mathbb{P}^{n}$. If $\Phi$ belongs to $J$ thus (7.3) provides a realization of the membership, expressed in terms of the current $U$ and the Hefer forms.

\section{The fundamental principle}

Let $E_{1}$ and $E_{0}$ be trivial bundles, let $F$ be a $\operatorname{Hom}\left(E_{1}, E_{0}\right)$-valued polynomial of generic rank $r_{0}=\operatorname{rank} E_{0}$ and let $F^{T}$ be the transpose of $F$. Furthermore, let $K$ be the closure of an open strictly convex bounded domain with smooth boundary in $\mathbb{R}^{n}$ containing the origin. The fundamental principle of Ehrenpreis and Palamodov states that every homogeneous solution to the system of equations $F^{T}(D) \xi=0, D=i \partial / \partial t$, on $K$ is a superposition of exponential solutions with frequencies in the algebraic set $Z=\{z ; \operatorname{rank} F(z)<r\}$. Following the ideas in [14] we can produce a residue version of the fundamental principle.

Let $\rho(\eta)$ be the support function $\sup _{t \in K}\langle\eta, t\rangle$ for $K$ but smoothened out in a neighborhood of the origin in $\mathbb{R}^{n}$. Since $\rho$ is smooth in $\mathbb{R}^{n}$ and 1-homogeneous outside a neighborhood of the origin, all its derivatives are bounded. Let

$$
\rho^{\prime}(\eta)=\left(\partial \rho / \partial \eta_{1}, \ldots, \partial \rho / \partial \eta_{n}\right)
$$

We extend to complex arguments $\zeta=\xi+i \eta$ by letting $\rho(\zeta)=\rho(\eta)$ and $\rho^{\prime}(\zeta)=\rho^{\prime}(\eta)$. Then $\rho^{\prime}$ maps $\mathbb{C}^{n}$ onto $K$, see [14]. The convexity of $\rho$ implies that

$$
e^{\rho(\zeta)}\left|e^{i\left\langle\rho^{\prime}(\zeta), \zeta-z\right\rangle}\right| \leqslant e^{\rho(z)}
$$

$4^{e}$ SÉRIE - TOME $40-2007-\mathrm{N}^{\circ} 6$ 
We are to modify the decomposition (7.3) to allow entire functions $h$ with values in $E_{0}$ satisfying an estimate like

$$
|h(z)| \leqslant C(1+|z|)^{M} e^{\rho(z)}
$$

for some, from now on, fixed natural number $M$. We will use the same notation as in the previous section. First we introduce a new weight.

LEMMA 8.1. - The form

$$
g^{\prime}=e^{i\left\langle\rho^{\prime}(\zeta), \zeta-z\right\rangle+\frac{i}{\pi} \partial \bar{\partial} \rho}=e^{i\left\langle\rho^{\prime}(\zeta), \zeta-z\right\rangle} \sum_{\ell \geqslant 0}\left(\frac{i}{\pi} \partial \bar{\partial} \rho\right)^{\ell} / \ell !
$$

is a weight for each fixed $z \in \mathbb{C}^{n}$.

Proof. - Since $\partial \rho / \partial \zeta_{k}=-(i / 2) \rho_{k}^{\prime}(\zeta)$,

$$
\gamma=i\left\langle\rho^{\prime}(\zeta), \zeta-z\right\rangle+\frac{i}{\pi} \partial \bar{\partial} \rho(\zeta)=\nabla_{\zeta-z} \frac{-\partial \rho}{\pi i}
$$

is $\nabla_{\zeta-z}$-closed and $\gamma_{0,0}(z)=0$. Thus $\gamma$ and $e^{\gamma}$ are weights.

It follows from (8.1) that

$$
g^{\mu} \wedge g^{\prime} \wedge H^{1} U h, \quad g^{\mu} \wedge g^{\prime} \wedge H^{0} R h
$$

will vanish to a given finite order at infinity if $\mu$ is large enough and $h(\zeta)$ satisfies (8.2). Therefore, if $\mu$ is large enough, using the compactly supported weights $g_{k}$ and arguing as in the proof of Theorem 7.2, we obtain the decomposition

$$
h(z)=F(z) \int g^{\prime} \wedge g^{\mu} \wedge H^{1} U h+\int g^{\prime} \wedge g^{\mu} \wedge H^{0} R h=F T h+S h
$$

for all entire $h$ satisfying (8.2). Furthermore, $S h$ vanishes if $h=F q$ for some holomorphic $q$, and in view of (8.1), both $T h$ and $S h$ satisfy (8.2) for some other large number $M^{\prime}$ instead of $M$.

Let $\mathcal{E}^{\prime}(K)$ be the space of distributions in $\mathbb{R}^{n}$ with support contained in $K$ and let $\mathcal{E}^{\prime}, M(K)$ denote the subspace of distributions of order at most $M$. For $\omega \in \mathcal{E}^{\prime}(K)$ let $\hat{\omega}(\zeta)=\omega\left(e^{-i\langle\zeta \cdot \cdot\rangle}\right)$ be its Fourier-Laplace transform. The Paley-Wiener-Schwartz theorem, see [26] Thm. 7.3.1, states that if $\nu \in \mathcal{E}^{\prime}, M(K)$, then

$$
|\hat{\nu}(\zeta)| \leqslant C(1+|\zeta|)^{M} e^{\rho(\eta)}
$$

and conversely: if $h$ is an entire function that satisfies such an estimate then $h=\hat{\nu}$ for some $\nu \in \mathcal{E}^{\prime}(K)$.

From (8.3), applied to $\hat{\nu}$ for $\nu \in \mathcal{E}^{\prime}, M\left(K, E_{0}\right)$, we therefore get mappings

$$
\mathcal{T}: \mathcal{E}^{\prime}, M\left(K, E_{0}\right) \rightarrow \mathcal{E}^{\prime}\left(K, E_{1}\right), \quad \mathcal{S}: \mathcal{E}^{\prime}, M\left(K, E_{0}\right) \rightarrow \mathcal{E}^{\prime}\left(K, E_{0}\right),
$$

such that

$$
\nu=F(-D) \mathcal{T} \nu+\mathcal{S} \nu
$$


and $\mathcal{S} \nu=0$ if $\nu=F(-D) \omega$ for some $\omega \in \mathcal{E}^{\prime}\left(K, E_{1}\right)$. By duality we have mappings

$$
\mathcal{T}^{*}: \mathcal{E}\left(K, E_{1}^{*}\right) \rightarrow C^{M}\left(K, E_{0}^{*}\right), \quad \mathcal{S}^{*}: \mathcal{E}\left(K, E_{0}^{*}\right) \rightarrow C^{M}\left(K, E_{0}^{*}\right)
$$

and they satisfy

$$
\xi=\mathcal{T}^{*} F^{T}(D) \xi+\mathcal{S}^{*} \xi, \quad \xi \in \mathcal{E}\left(K, E_{0}^{*}\right) .
$$

THEOREM 8.2. - Suppose that $M \geqslant \operatorname{deg} F$. If $\xi \in \mathcal{E}\left(K, E_{0}^{*}\right)$, then $\mathcal{S}^{*} \xi \in C^{M}\left(K, E_{0}^{*}\right)$ satisfies $F^{T}(D) \mathcal{S}^{*} \xi=0$. If in addition $F^{T}(D) \xi=0$, then $S^{*} \xi=\xi$. Moreover, we have the explicit formula

$$
\mathcal{S}^{*} \xi(t)=\int_{\zeta} R^{T}(\zeta) \alpha^{T}(\zeta, D) \xi\left(\rho^{\prime}\right) e^{-i\left\langle\zeta, t-\rho^{\prime}\right\rangle} \wedge e^{\frac{i}{\pi} \partial \bar{\partial} \rho}
$$

where $\alpha^{T}(\zeta, D) \xi\left(\rho^{\prime}\right)$ is the result when replacing each occurrence of $z$ in $\alpha^{T}(\zeta, z)$ by $D$, letting it act on $\xi(t)$ and evaluating at the point $\rho^{\prime}(\zeta)$.

Thus $\mathcal{S}^{*}$ is a projection onto the space of homogeneous solutions.

Recall that $\rho^{\prime} \in K$. Also notice that $\operatorname{Re}-i\langle\zeta, t\rangle=\langle\eta, t\rangle \leqslant \rho(\eta)$ if $t \in K$, so combined with (8.1) we get that

$$
\operatorname{Re}-i\left\langle\zeta, t-\rho^{\prime}(\zeta)\right\rangle \leqslant 0, \quad t \in K
$$

(for $\zeta$ outside a neighborhood of 0 ). Therefore the integral in (8.6) has meaning if $\mu$ is large enough.

Proof. - Suppose that $M \geqslant \operatorname{deg} F$. Then for $\omega \in \mathcal{E}^{\prime}, M-\operatorname{deg} F\left(K, E_{1}\right)$ we have

$$
\omega \cdot F^{T}(D) \mathcal{S}^{*} \xi=F(-D) \omega \cdot \mathcal{S}^{*} \xi=\mathcal{S}(F(-D) \omega) \cdot \xi=0
$$

since $\tau=F(-D) \omega \in \mathcal{E}^{\prime}, M\left(K, E_{0}\right)$ so that $\mathcal{S} \tau=0$. From (8.7) the first statement now follows. The second one follows immediately from (8.5).

It remains to prove (8.6). The argument is very similar to the proof of Theorem 2 in [14] so we only sketch it. To begin with we have

$$
S \hat{\nu}(z)=\int_{\zeta} \alpha(\zeta, z) R(\zeta) \hat{\nu}(\zeta) e^{i\left\langle\zeta-z, \rho^{\prime}(\zeta)\right\rangle} \wedge e^{\frac{i}{\pi} \partial \bar{\partial} \rho}
$$

where $\alpha(\cdot, z)=g^{\mu} \wedge H^{0}$ is a polynomial in $z$. Let $\delta_{t}$ be the Dirac measure at $t \in K$. Then, letting $T$ denote transpose of matrices, we have

$$
\begin{aligned}
\mathcal{S}^{*} \xi(t) & =\delta_{t} \cdot \mathcal{S}^{*} \xi=\left(\mathcal{S} \delta_{t} \cdot \xi\right)^{T} \\
& =\frac{1}{(2 \pi)^{n}} \int_{x} \int_{\zeta} R^{T}(\zeta) \alpha^{T}(\zeta, x) e^{-i\left\langle x, \rho^{\prime}\right\rangle} \hat{\xi}(-x) e^{-i\left\langle\zeta, t-\rho^{\prime}\right\rangle} \wedge e^{\frac{i}{\pi} \partial \bar{\partial} \rho} .
\end{aligned}
$$

As in [14] one can verify that it is legitimate to interchange the order of integration, and then (8.6) follows by Fourier's inversion formula.

$4^{\text {e }}$ SÉRIE - TOME $40-2007-\mathrm{N}^{\circ} 6$ 
COROLlaRY 8.3. - For any solution $\xi \in \mathcal{E}\left(K, E_{0}^{*}\right)$ of $F^{T}(D) \xi=0$, there are smooth forms $A_{k}(\zeta)$ with values in $E_{k}^{*}$ such that

$$
\xi(t)=\int_{\zeta} \sum_{k} R_{k}^{T}(\zeta) A_{k}(\zeta) e^{-i\left\langle\zeta, t-\rho^{\prime}(\zeta)\right\rangle} .
$$

Conversely, for any such smooth forms $A_{k}(\zeta)$ with sufficient polynomial decay at infinity the integral (8.9) defines a homogeneous solution.

The last statement follows just by applying $F^{T}(D)$ to the integral and using that $F^{T}(\zeta) R^{T}=0$.

Remark 4. - In case $F$ defines a complete intersection, formulas similar to (8.9) were obtained in [14] and [32]. In [14] is assumed, in addition, that $F^{T}(D)$ is hypoelliptic; then one can avoid the polynomial weight factor $g^{\mu}$ and so the resulting formula is even simpler. See also [10] and [12].

Example 6 (A final example). - The ideal $\left(z_{1}^{2}, z_{1} z_{2}\right)$ corresponds to the system

$$
\frac{\partial^{2}}{\partial t_{1}^{2}} \xi(t)=0, \quad \frac{\partial^{2}}{\partial t_{1} \partial t_{2}} \xi(t)=0
$$

In view of (8.9) and Example 2, the solutions are precisely the functions that can be written

$$
\begin{aligned}
\xi(t)= & \int_{z}\left[\frac{1}{z_{2}}\right] \bar{\partial}\left[\frac{1}{z_{1}}\right] \wedge A_{1}(z) d z_{2} \wedge d \bar{z}_{1} \wedge d \bar{z}_{2} e^{-i\left(z_{1} t_{1}+z_{2} t_{2}\right)} \\
& +\int_{z} \bar{\partial}\left[\frac{1}{z_{1}^{2}}\right] \wedge \bar{\partial}\left[\frac{1}{z_{2}}\right] \wedge A_{2}(z) d \bar{z}_{1} \wedge d \bar{z}_{2} e^{-i\left(z_{1} t_{1}+z_{2} t_{2}\right)},
\end{aligned}
$$

for smooth functions $A_{1}$ and $A_{2}$ with appropriate growth. It is easily checked directly to be the general solution, since the first integral is a quite arbitrary function $C\left(t_{2}\right)$ whereas the second integral is an arbitrary polynomial $C_{1}+C_{2} t_{1}$.

\section{Acknowledgement}

We express our sincere gratitude to Jan-Erik Björk, Ralf Fröberg, and Alain Yger for invaluable discussions on these matters. We also thank the referee for several suggestions for improvements of the presentation.

\section{REFERENCES}

[1] Andersson M., Residue currents and ideals of holomorphic functions, Bull. Sci. Math. 128 (2004) 481-512.

[2] Andersson M., Ideals of smooth functions and residue currents, J. Funct. Anal. 212 (1) (2004) 76-88.

[3] Andersson M., The membership problem for polynomial ideals in terms of residue currents, Ann. Inst. Fourier 56 (2006) 101-119.

[4] ANDERSSON M., Residue currents of holomorphic morphisms, J. reine angew. Math. 596 (2006) 215234. 
[5] AndersSON M., Integral representation with weights II, division and interpolation formulas, Math. Z. 254 (2006) 315-332.

[6] BAYER D., MUMFord D., What can be computed in algebraic geometry?, in: Computational Algebraic Geometry and Commutative Algebra, Cortona, 1991, in: Sympos. Math., vol. XXXIV, Cambridge Univ. Press, Cambridge, 1993, pp. 1-48.

[7] Bayer D., Peeva I., Sturmfels B., Monomial resolutions, Math. Res. Lett. 5 (1-2) (1998) 31-46.

[8] BAyer D., Stillman M., A criterion for detecting $m$-regularity, Invent. Math. 87 (1987) 1-11.

[9] BAyer D., Sturmfels B., Cellular resolutions of monomial modules, J. reine angew. Math. 502 (1998) 123-140.

[10] Berenstein C., Yger A., About L. Ehrenpreis fundamental principle, in: Geometrical and Algebraical Aspects in Several Complex Variables, Cetraro, 1989, in: Sem. Conf., vol. 8, EditEl, Rende, 1991, pp. 47-61.

[11] Berenstein C., Yger A., Effective Bezout identities in $Q\left[z_{1}, \ldots, z_{n}\right]$, Acta Math. 166 (1991) 69120.

[12] Berenstein C., GAy R., Vidras A., Yger A., Residue Currents and Bézout Identities, Birkhäuser, 1993.

[13] Berndtsson B., A formula for division and interpolation, Math. Ann. 263 (1983).

[14] Berndtsson B., Passare M., Integral formulas and an explicit version of the fundamental principle, J. Funct. Anal. 84 (1989).

[15] BJÖRK J.-E., Residues and D-modules, in: The legacy of Niels Henrik Abel, Springer, Berlin, 2004, pp. 605-651.

[16] ColefF N.R., Herrera M.E., Les courants résiduels associés à une forme méromorphe, Lect. Notes in Math., vol. 633, Springer-Verlag, Berlin-Heidelberg-New York, 1978.

[17] Demailly J.P., Complex Analytic and Differential Geometry Monograph Grenoble, 1997.

[18] Dickenstein A., Sessa C., Canonical representatives in moderate cohomology, Invent. Math. 80 (1985) 417-434.

[19] Dickenstein A., Gay R., Sessa C., Yger A., Analytic functionals annihilated by ideals, Manuscripta Math. 90 (1996) 175-223.

[20] Ehrenpreis L., Fourier Analysis in Several Complex Variables, Pure and Applied Mathematics, vol. XVII, Wiley-Interscience Publishers. A Division of John Wiley and Sons, New York-LondonSydney, 1970.

[21] Eisenbud D., Commutative Algebra. With a View Toward Algebraic Geometry, Graduate Texts in Mathematics, vol. 150, Springer-Verlag, New York, 1995.

[22] Eisenbud D., The Geometry of Syzygies. A Second Course in Commutative Algebra and Algebraic Geometry, Graduate Texts in Mathematics, vol. 229, Springer-Verlag, New York, 2005.

[23] Griffiths Ph., Harris J., Principles of Algebraic Geometry, John Wiley and Sons, 1978.

[24] Gunning R., Rossi H., Analytic Functions of Several Complex Variables, Prentice-Hall Inc., Englewood Cliffs, NJ, 1965.

[25] HöRmAnder L., An Introduction to Complex Analysis in Several Variables, Second revised edition, North-Holland Mathematical Library, vol. 7, North-Holland Publishing Co., American Elsevier Publishing Co., Inc., Amsterdam-London/New York, 1973.

[26] HöRMAnder L., The Analysis of Linear Partial Differential Operators I, second ed., Springer-Verlag, 1990.

[27] MAYR E., MAYER A., The complexity of the word problem for commutative semigroups and polynomial ideals, Adv. Math. 46 (1982) 305-329.

[28] Miller E., Sturmfels B., Combinatorial Commutative Algebra, Graduate Texts in Mathematics, vol. 227, Springer-Verlag, New York, 2005.

[29] Nöther M., Über einen Satz aus der Theorie der algebraischen Functionen, Math. Ann. (1873) 351359.

[30] Palamodov V.P., Linear Differential Operators with Constant Coefficients, Translated from the Russian by A.A. Brown. Die Grundlehren der mathematischen Wissenschaften, vol. 168, SpringerVerlag, New York-Berlin, 1970.

[31] Passare M., Residues, currents, and their relation to ideals of holomorphic functions, Math. Scand. 62 (1988) 75-152.

$4^{\mathrm{e}}$ SÉRIE - TOME $40-2007-\mathrm{N}^{\circ} 6$ 
[32] YGer A., Formules de division et prolongement méromorphe, in: Séminaire d'Analyse P. LelongP. Dolbeault-H. Skoda, Années 1985/1986, in: Lecture Notes in Math., vol. 1295, Springer, Berlin, 1987, pp. 226-283.

[33] Passare M., Tsikh A., Yger A., Residue currents of the Bochner-Martinelli type, Publ. Mat. 44 (2000) 85-117.

[34] QUILLEN D., Superconnections and the Chern character, Topology 24 (1985) 89-95.

[35] ShiffMan B., Degree bounds for the division problem in polynomial ideals, Michigan Math. J. 36 (1989) 163-171.

[36] Wulcan E., Residue currents of monomial ideals, Indiana Univ. J. 56 (2007) 36-388.

[37] WulCAN E., Residue currents and their annihilator ideals, Thesis Gothenburg, 2007.

(Manuscrit reçu le 3 juillet 2007;

accepté, après révision, le 12 novembre 2007.)

Mats ANDERSSON

Department of Mathematics,

Chalmers University of Technology and the

University of Göteborg,

S-412 96 Göteborg, Sweden

E-mail: matsa@math.chalmers.se

Elizabeth WULCAN

Department of Mathematics,

Chalmers University of Technology and the

University of Göteborg,

S-412 96 Göteborg, Sweden

E-mail: wulcan@math.chalmers.se 